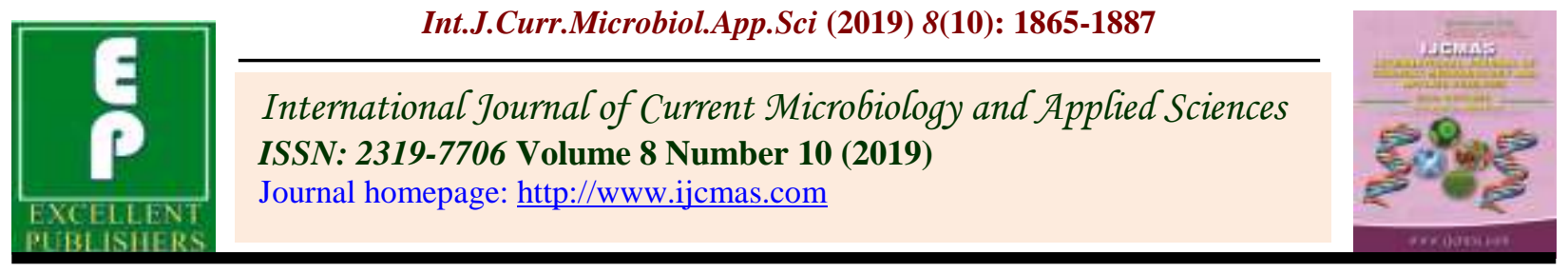

Original Research Article

https://doi.org/10.20546/ijcmas.2019.810.217

\title{
PGR Diversity and Economic Utilization of Orchids
}

\author{
R. K. Pamarthi, R. Devadas, Raj Kumar, D. Rai, P. Kiran Babu, \\ A. L. Meitei, L. C. De, S. Chakrabarthy, D. Barman and D. R. Singh* \\ ICAR-NRC for Orchids, Pakyong, Sikkim, India \\ ICAR-IARI, Kalimpong, West Bengal, India \\ *Corresponding author
}

\begin{tabular}{|l|}
\hline Ke y w o r d s \\
Orchids, Collection, \\
$\begin{array}{l}\text { Conservation, } \\
\text { Utilization }\end{array}$ \\
\hline Article Info \\
\hline $\begin{array}{l}\text { Accepted: } \\
\text { 15 September } 2019 \\
\text { Available Online: } \\
10 \text { October } 2019\end{array}$ \\
\hline
\end{tabular}

\section{Introduction}

Orchidaceae is one of the most ecologically and morphologically diverse families of flowering plants. It is the second largest family of flowering plants in the world, comprising of nearly 800 genera and 22,500 species (Mabberley, 2008; Meitei, 2019; Singh, 2019). Orchids are one such group of plants which grow in a variety of habitats throughout the globe, but they are very sensitive to habitat change. Understanding the prospects, horticultural and medicinal value, and the family is gaining much attention throughout the world to unfold the biology, evolution, taxonomy, cytology, chemistry, hybridization and cultivation etc. In North East India, many orchids are used for different 
purposes such as ornamentals, medicine, food, also in socio-cultural events (Deb and Imchen, 2008; Deb, 2009; Medhi and Chakrabarti, 2009). Orchids are used as cut flowers, bouquet, loose flowers, dried flowers, as single flower mountings, potted plant display, in perfumery industries and by products in handicrafts (Singh, 2019).

ICAR- NRC for Orchids, Pakyong exclusively working on orchid breeding as well as production of quality planting materials which are considered as major bottlenecks in the development of orchid industry (Meitei et al., 2019). This institute plays a crucial role for with an aim that of research was on collection, characterization, evaluation, conservation conserving the indigenous orchid resources and utilization of genetic resources available in the country particularly in north-eastern region. And carry out research in identification, isolation, characterization of novel genes from native species, development of hybrids for commercial cultivation. And the institute is one of the National Active Germplasm Sites (NAGS) units in the Indian Plant genetic resource information system. This paper aims that the status of collection and conservation, utilization of indigenous orchid germplasm resources at this institute.

\section{Materials and Methods}

Several exploration programmes were carried since 1996 for collecting the valuable orchid germplasm various parts of the country (Fig. 1). Structural and well planned explorations conducted in the orchid rich biodiversity hotspots. Plant material in the form of plantlets, bulbs, capsule fruits, seeds and floral parts were collected and these plant materials was acclimatized and conserved in ICARNRCO polyhouses and used for breeding programmes. The collected orchids were identified up to the species to tribe level with the help of published literature such as,
Hooker (1888-1890), Blatter (1928), Pradhan (1976), Bose and Bhattacharjee (1980), Rao (1998), Ahmedullah and Nayar (1987), Ansari and Balakrishnan (1990), Kumar and Manilal (1994), Lakshminarasimhan (1996), Nayar (1996), Karthikeyan (2000), Singh (2001), Pearce and Cribb (2002) Mishra (2007), Lucksom (2007, 2011), Mabberley (2008) Rampal and Singh (2016) and Singh (2019). The online databases, namely, Govaerts (2012) http://apps. Kew.org/wcsp, Tropicos (2018) www.tropicos.org, IPNI (2018) www.ipni.org, eFloras www.efloras.org, www.theplantlist.org (2018) were also consulted for recent updates on the plant names and distribution. The species were confirmed with the help of herbaria of The Botanical Survey of India, (CAL) and Regional Centre (BHSC), Gangtok, Sikkim, Sikkim University, Sikkim (SKM) and were visited and data on habitat, locality, altitude and flowering were gathered. Based on this information past localities from where the species were collected were also visited, to know the present status and changes in population-size if any. The RET orchid species are listed based on IUCN red list category, the economic importance and mode of utilization of the species such as medicinal, dietary and breeding purposes are also provided (Table 1). Ex-situ conservation of orchids in the institute poly houses and new techniques developed for conservation of orchids through indigenous technologies and DNA barcoding for few species were carried.

\section{DNA barcoding of Indian orchids}

The conservation of orchids is carried out considering their status in the habitat. The basis of conservation is laid on the basis of certain objectives such as conservation of threatened species, Molecular approaches, such as DNA-based methods have transformed understanding and appreciation of conservation issues associated with orchids. 
Molecular data provide an empirical framework through which conservation practitioners are in a more informed position to define priorities, reduce costs and optimize management decisions (Zaman, 1998). In particular, molecular data enable conservationists to address questions of genetic variation within and between populations, species or provenance delimitation and the maintenance of evolutionary processes (Fay and Krauss, 2003).

\section{Results and Discussion}

A total of 351 species from 94 genera were collected through several explorations carried across the country. Among the collections, 205 species were categorized as threatened species, followed by 90 species having breeding value, 87 species which are used in traditional medicine, 77 species having fragrance and 11 species were used in traditional dietary (Table 1). The genus Dendrobium representing highest number of species (68), followed by Bulbophyllum (30), Cymbidium (21), Coelogyne (19), Calanthe (12), Liparis (11), Vanda (10), Eria (9), Pinalia (8), Paphiopedilum (7), Aerides, Gastrochilus, Oberonia, Pholidota (6), Cleistostoma, Goodyera (5), Luisia, Papilonanthe, Phalaenopsis, Pleione, Sunipia (3), Agrostophyllum, Crepidium, Epidendrum, Micropera, Otochilus, Phaius, Thunia,Zeuxine (3), Acampe, Ascocentrum, Callostylis, Ceratostylis, Cryptochilus, Esmeralda, Habenaria, Herminium, Panisea, Phreatia, Thelasis and 45 genus representing single species respectively (Fig. 1-5).

\section{Importance of orchids}

\section{Breeding value}

Plant Breeders carry out the hybridization program based on various objectives such as better flower colour, shape and size, length of inflorescence, increasing number of flowers, producing miniature forms, blooming season and period, fragrant harbouring, suitable potted plants, hybrids for different temperature and light regimes; resistant lines for biotic and abiotic stresses etc. A total of 90 species having potential breeding value which was conserved at the institute in the following genera viz., Calanthe, Cymbidium, Dendrobium, Phalaenopsis, Cattleya, Oncidium, Platanthera, Masdevallia, Paphiopedilum and Vanda were utilized in breeding programmes to develop hybrids or improve lines. Subsequent milestones have been marked in the field of hybrid development throughout the globe using modern technologies and approaches. Extensive researches viz. species compatibility, apomixis, genetic engineering, mutation breeding, ploidy breeding etc., have been done by utilizing the natural species and hybrids in the orchid improvement programmes. Populations were developed viz., intergeneric combination (1 no.), primary species hybrid combinations (5 no.), secondary hybrid (10 no.), tertiary hybrid combinations (11 no.) and somaclonal mutants ( 2 no.) using existing germplasm collections. Of which, 18 breeding lines/ genetic stocks are registered with national register for IC numbers with national gene back ICARNBPGR, New Delhi and 45 breeding lines were submitted. The State flower of Mizoram and endangered species, Renanthera imschootiana known as 'Red Vanda' was registered with NBPGR (IC 566525/INGR 10113) for floral characters in 2010. In the year 2012, the breeding cycle of orchids was re-invented in India after the flowering of primary hybrid from first indigenous cross, PBX-05-56/2012 (C. lowianum x C. tigrinum), which was earlier bred by R.I. Measures in 1903 from United Kingdom. Two scented lines PBX-05-772 ad PBX-05-751 were developed by 2013 using native scented 
species, Cymbidium iridiodes as male parent. The three varieties viz., 'B. $\mathrm{S}$. Basnet'(Cymbidium) from $C$. lowianum x $C$. tigrinum, 'Kunga Gyatso' (Aranda) from (Arachnis clarkii x Vanda coerulea) and 'V. Nagaraju' (Dendrobium) were identified at institution level for cultivation based on VCU basis. In 2016, Paphiopedilum variety (Lady's Slipper Orchid) 'Sheetal 1' (IC 614753) was filed with PPVFRA, New Delhi for legal protection and received acknowledgement (No: REG/2016/1534 date: 16.09.2016). Cross variability in Moth Orchid (Phalaenopsis) breeding lines (PBX-12-99) derived exotic varieties as parents was established during 2016. Promising Vanda breeding lines (PBX12-169) using native bred species with commercial hybrid resulted from cross breeding was established by 2017 .

Behavior pattern of Asiatic Dendrobium species at both intra and inter-sectional compatibility at species level and with modern hybrids was established and reported (Devadas 2016). High compatibility nature among Indian Cymbidium species and as well as with modern Cymbidium hybrids was reported (Devadas, 2013, 2014) and Vanda species was proven (in press). High level of incompatibility was observed Phaius genera with other Orchid genera, like similar sympodial orchids like Calanthe, Coelogyne, Phalaenopsis, Lycaste, Dienia, Cymbidium, Thunia, Paphiopedilum, Coelogyne, Eria and monopodial orchids like Paplionanthe, Dendrobium, Arundina and Vanda. However, primary species hybrid was made with Phaius using native species in both direct (PBX-1122) and reciprocal combinations (PBX-11-25), where the natural hybrid was never reported earlier (Devadas, 2019).

\section{Socio-cultural importance}

The beautiful fox-tail orchid (Rhynchostylis retusa) locally called 'Kopou Phul'in Assam is worn by ladies on their head as ornament during different festival especially during 'Bihu' festival in Assam. It symbolizes youthfulness during springtime a symbol of love by the youth of the Ahom community (Deb 2013; Medhi and Chakrabarti, 2009). In Nagaland, Dendrobium hookerianum, Dendrobium nobilesymbolizes purity and holiness. Dendrobium acinaforme plant is worn by the head hunting community with the belief giving courage and good luck in their hunt (Deb and Imchen, 2008). In Manipur (erstwhile Kangleipak), orchids origination are defined in many historic mentioned and ceremony. The flowers of orchids such as Vanda tessellata and Coelogyne nitida are used during local festivals in Assam and Arunachal Pradesh and Papilionanthe teres flowers are used by the Tai ethnics of Assam and Arunachal Pradesh for offerings to Lord Budha and spirits (Medhi and Chakrabarti, 2009). This species are conserved and used in breeding programmes at this institute.

\section{Orchids used in medicine}

The medicinal value of orchids is found to be recorded as early as 250 - 300 B.C. by Susruta and Vagbhata respectively, from ancient Sanskrit. Many orchids are used in traditional medicine treatment as a remedy for several ailments since ancient times. Orchid genera which were used for medicinal purpose Medicinal orchids mainly belong to genera: Calanthe, Coelogyne, Cymbidium, Cypipedium, Dendrobium, Ephemerantha, Eria, Galeola, Gastrodia, Gymnadenia, Habenaria, Ludisia, Luisia, Nevilia and Thunia (Gutierrez, 2010). In India, some orchids like Eulophia campestris, Orchis latifolia, Vanda roxburgii have drawn the attention of scientific community because of their medicinal properties (Singh et al., 2009). Dendrobium macraei and $D$. nobile are another important orchids from Ayurvedic point of view as it is reported to be source of Jivant (Kasera 2001; Meitei 2019). 
Table.1 List of orchid species collected and conserved at ICAR-NRC for orchids

\begin{tabular}{|c|c|c|c|c|c|c|c|}
\hline $\begin{array}{c}\text { S. } \\
\text { No. }\end{array}$ & Botanical Name & $\begin{array}{c}\text { RET } \\
\text { status }\end{array}$ & $\begin{array}{c}\text { Medicinal } \\
\text { value }\end{array}$ & \begin{tabular}{|c|} 
Dietary \\
suppliment
\end{tabular} & Fragrant & $\begin{array}{c}\text { Breeding } \\
\text { value }\end{array}$ & Remarks \\
\hline 1 & $\begin{array}{l}\text { Acampe praemorsa (Roxb.) Blatt. and } \\
\text { McCann }\end{array}$ & & $\sqrt{ }$ & & $\sqrt{ }$ & & \\
\hline 2 & Acampe rigida (Buch.-Ham. ex Sm.) P.F.Hunt & $\sqrt{ }$ & $\sqrt{ }$ & & $\sqrt{ }$ & & \\
\hline 3 & Acanthephippium sylhetense Lindl. & $\sqrt{ }$ & & & $\sqrt{ }$ & & \\
\hline 4 & Acrochaene punctata Lindl. & $\sqrt{ }$ & & & & & \\
\hline 5 & Aerides crispa Lindl. & $\sqrt{ }$ & & & & & \\
\hline 6 & Aerides maculosa Lindl. & $\sqrt{ }$ & & & $\sqrt{ }$ & & Endemic \\
\hline 7 & Aerides multiflora Roxb. & & $\sqrt{ }$ & & $\sqrt{ }$ & & \\
\hline 8 & Aerides odorata Lour. & & $\sqrt{ }$ & & $\sqrt{ }$ & & \\
\hline 9 & Aerides rosea Lodd. ex Lindl. and Paxton & $\sqrt{ }$ & $\sqrt{ }$ & & $\sqrt{ }$ & & \\
\hline 10 & Agrostophyllum brevipes King and Pantl. & & $\sqrt{ }$ & & & & \\
\hline 11 & Agrostophyllum callosum Rchb.f. & & $\sqrt{ }$ & & & & \\
\hline 12 & $\begin{array}{l}\text { Agrostophyllum planicaule (Wall. ex Lindl.) } \\
\text { Rchb.f. }\end{array}$ & & & & & & \\
\hline 13 & Anthogonium gracile Wall. ex Lindl. & & $\sqrt{ }$ & & & & \\
\hline 14 & $\begin{array}{l}\text { Anthogonium gracile Wall. ex Lindl. (White } \\
\text { variant) }\end{array}$ & & & & & & Variant \\
\hline 15 & Arachnis labrosa (Lindl. and Paxton) Rchb.f. & & & & & $\sqrt{ }$ & \\
\hline 16 & Arundina graminifolia (D.Don) Hochr. & $\sqrt{ }$ & $\sqrt{ }$ & & & & \\
\hline 17 & Ascocentrum ampullaceum (Roxb.) Schltr. & $\sqrt{ }$ & & & & $\sqrt{ }$ & \\
\hline 18 & Ascocentrum aurantiacum Schltr. & & & & & $\sqrt{ }$ & \\
\hline 19 & Bulbophyllum affine Wall. ex Lindl. & $\sqrt{ }$ & & & & & \\
\hline 20 & Bulbophyllum careyanum (Hook.) Spreng. & $\sqrt{ }$ & $\sqrt{ }$ & & & & \\
\hline 21 & Bulbophyllum caudatum Lindl. & & & & & & \\
\hline 22 & Bulbophyllum cauliflorum Hook. f. & $\sqrt{ }$ & & & & & \\
\hline 23 & Bulbophyllum crassipes Hook.f. & $\sqrt{ }$ & & & & & \\
\hline 24 & Bulbophyllum cylindraceum Wall. ex Lindl. & $\sqrt{ }$ & & & & & \\
\hline 25 & Bulbophyllum eublepharum Rchb.f. & $\sqrt{ }$ & & & & & \\
\hline 26 & Bulbophyllum fimbriatum (Lindl.) Rchb.f. & & & & & & \\
\hline 27 & Bulbophyllum fischeri Seidenf. & $\sqrt{ }$ & & & & & \\
\hline 28 & $\begin{array}{l}\text { Bulbophyllum guttulatum (Hook.f.) } \\
\text { N.P.Balakr. }\end{array}$ & $\sqrt{ }$ & & & & & \\
\hline 29 & Bulbophyllum gymnopus Hook.f. & $\sqrt{ }$ & & & & & \\
\hline 30 & Bulbophyllum hirtum (Sm.) Lindl. ex Wall. & $\sqrt{ }$ & & & $\sqrt{ }$ & & \\
\hline 31 & $\begin{array}{l}\text { Bulbophyllum interpositum J.J.Verm., Schuilt. } \\
\text { and de Vogel }\end{array}$ & & & & & & \\
\hline
\end{tabular}




\begin{tabular}{|c|c|c|c|c|c|c|}
\hline 32 & $\begin{array}{l}\text { Bulbophyllum jejosephii J.J.Verm., Schuit. } \\
\text { and de Vogel }\end{array}$ & & & & & Endemic \\
\hline 33 & $\begin{array}{l}\text { Bulbophyllum leopardinum (Wall.) Lindl. ex } \\
\text { Wall. }\end{array}$ & $\sqrt{ }$ & $\sqrt{ }$ & $\sqrt{ }$ & $\sqrt{ }$ & \\
\hline 34 & Bulbophyllum mysorense (Rolfe) J.J.Sm. & $\sqrt{ }$ & & & & Endemic \\
\hline 35 & Bulbophyllum nodosum (Rolfe) J.J.Sm. & $\sqrt{ }$ & & & & Endemic \\
\hline 36 & $\begin{array}{l}\text { Bulbophyllum odoratissimum (Sm.) Lindl. ex } \\
\text { Wall. }\end{array}$ & $\sqrt{ }$ & $\sqrt{ }$ & $\sqrt{ }$ & & \\
\hline 37 & Bulbophyllum polyrrhizum Lindl. & $\sqrt{ }$ & & & & \\
\hline 38 & Bulbophyllum protractum Hook.f. & $\sqrt{ }$ & & & & \\
\hline 39 & Bulbophyllum refractum (Zoll.) Rchb.f. & & & & & \\
\hline 40 & Bulbophyllum repens Griff. & $\sqrt{ }$ & & & & \\
\hline 41 & Bulbophyllum reptans (Lindl.) Lindl. ex Wall. & $\sqrt{ }$ & & & & \\
\hline 42 & Bulbophyllum rigidum King and Pantl. & $\sqrt{ }$ & & & & \\
\hline 43 & Bulbophyllum roxburghii (Lindl.) Rchb.f. & $\sqrt{ }$ & & & & \\
\hline 44 & $\begin{array}{l}\text { Bulbophyllum sarcophyllum (King and Pantl.) } \\
\text { J.J.Sm. }\end{array}$ & & & & & \\
\hline 45 & Bulbophyllum sterile (Lam.) Suresh & & $\sqrt{ }$ & & & Endemic \\
\hline 46 & Bulbophyllum striatum (Griff.) Rchb.f. & $\sqrt{ }$ & & & & \\
\hline 47 & $\begin{array}{l}\text { Bulbophyllum trichocephalum (Schltr.) } \\
\text { Tang\&F.T.Wang var trichocephalum }\end{array}$ & & & & & \\
\hline 48 & Bulbophyllum triste Rchb.f. & $\sqrt{ }$ & & & & \\
\hline 49 & Bulbophyllum umbellatum Lindl. & & & & & \\
\hline 50 & Bulbophyllum viridiflorum (Hook.f.) Schltr. & $\sqrt{ }$ & & & & \\
\hline 51 & Bulbophyllum wallichii Rchb.f. & $\sqrt{ }$ & & & & \\
\hline 52 & Bulleyia yunnanensis Schltr. & $\sqrt{ }$ & & & & \\
\hline 53 & Calanthe biloba Lindl. & $\sqrt{ }$ & & & & \\
\hline 54 & Calanthe brevicornu Lindl. & $\sqrt{ }$ & & & $\sqrt{ }$ & \\
\hline 55 & Calanthe chloroleuca Lindl. & & & & $\sqrt{ }$ & \\
\hline 56 & Calanthe davidii Franch. & $\sqrt{ }$ & & & $\sqrt{ }$ & \\
\hline 57 & Calanthe herbacea Lindl. & $\sqrt{ }$ & & & & \\
\hline 58 & Calanthe mannii Hook.f. & $\sqrt{ }$ & & & $\sqrt{ }$ & \\
\hline 59 & Calanthe plantaginea Lindl. & $\sqrt{ }$ & $\sqrt{ }$ & & & \\
\hline 60 & Calanthe puberula Lindl. & $\sqrt{ }$ & $\sqrt{ }$ & & $\sqrt{ }$ & \\
\hline 61 & Calanthe sylvatica (Thouars) Lindl. & $\sqrt{ }$ & $\sqrt{ }$ & & $\sqrt{ }$ & \\
\hline 62 & Calanthe triplicata (Willemet) Ames & $\sqrt{ }$ & & & $\sqrt{ }$ & \\
\hline 63 & Calanthe trulliformis King and Pantl. & & & & & \\
\hline 64 & Calanthe yuksomnensis Lucksom & $\sqrt{ }$ & & & $\sqrt{ }$ & Endemic \\
\hline 65 & $\begin{array}{l}\text { Callostylis bambusifolia (Lindl.) S.C.Chen } \\
\text { and J.J.Wood }\end{array}$ & $\sqrt{ }$ & $\sqrt{ }$ & & & \\
\hline 66 & Callostylis rigida Blume & & & & & \\
\hline
\end{tabular}




\begin{tabular}{|c|c|c|c|c|c|c|c|}
\hline 67 & $\begin{array}{l}\text { Cephalantheropsis obcordata (Lindl.) } \\
\text { Ormerod }\end{array}$ & & & & $\sqrt{ }$ & & \\
\hline 68 & Ceratostylis himalaica Hook.f. & & & & & & \\
\hline 69 & Ceratostylis subulata Blume & $\sqrt{ }$ & & & & & \\
\hline 70 & Chiloschista parishii Seidenf. & & & & & & \\
\hline 71 & Chrysoglossum ornatum Blume & $\sqrt{ }$ & & & & & \\
\hline 72 & $\begin{array}{l}\text { Cleisocentron pallens (Cathcart ex Lindl.) } \\
\text { N.Pearce and P.J.Cribb }\end{array}$ & & & & & & Endemic \\
\hline 73 & $\begin{array}{l}\text { Cleisostoma appendiculatum (Lindl.) Benth. } \\
\text { and Hook.f. ex B.D.Jacks. }\end{array}$ & $\sqrt{ }$ & & & & & \\
\hline 74 & Cleisostoma filiforme (Lindl.) Garay & & & & & & \\
\hline 75 & $\begin{array}{l}\text { Cleisostoma linearilobatum (Seidenf. and } \\
\text { Smitinand) Garay }\end{array}$ & & & & & & \\
\hline 76 & Cleisostoma subulatum Blume & & & & & & \\
\hline 77 & Cleisostoma tenuifolium (L.) Garay & & & & & $\sqrt{ }$ & \\
\hline 78 & Coelogyne barbata Lindl. ex Griff. & $\sqrt{ }$ & & & $\sqrt{ }$ & $\sqrt{ }$ & \\
\hline 79 & Coelogyne breviscapa Lindl & $\sqrt{ }$ & & & & $\sqrt{ }$ & \\
\hline 80 & Coelogyne corymbosa Lindl. & $\sqrt{ }$ & $\sqrt{ }$ & & $\sqrt{ }$ & $\sqrt{ }$ & \\
\hline 81 & Coelogyne cristata Lindl. & $\sqrt{ }$ & $\sqrt{ }$ & & $\sqrt{ }$ & & \\
\hline 82 & Coelogyne elata Lindl. & . & & & & & \\
\hline 83 & Coelogyne fimbriata Lindl. & & $\sqrt{ }$ & & & & \\
\hline 84 & Coelogyne flaccida Lindl. & $\sqrt{ }$ & $\sqrt{ }$ & & & $\sqrt{ }$ & \\
\hline 85 & Coelogyne fuscescens Lindl. & $\sqrt{ }$ & $\sqrt{ }$ & & & & \\
\hline 86 & Coelogyne hitendrae S.Das and S.K.Jain & $\sqrt{ }$ & & & & & Endemic \\
\hline 87 & Coelogyne longipes Lindl. & $\sqrt{ }$ & & & $\sqrt{ }$ & $\sqrt{ }$ & \\
\hline 88 & Coelogyne nitida (Wall. ex D.Don) Lindl. & $\sqrt{ }$ & $\sqrt{ }$ & & $\sqrt{ }$ & & \\
\hline 89 & Coelogyne occultata Hook.f. & $\sqrt{ }$ & & & $\sqrt{ }$ & & \\
\hline 90 & Coelogyne ovalis Lindl. & $\sqrt{ }$ & $\sqrt{ }$ & & $\sqrt{ }$ & & \\
\hline 91 & Coelogyne pempahisheyana H.J.Chowdhery & & & & & & \\
\hline 92 & Coelogyne prolifera Lindl. & $\sqrt{ }$ & $\sqrt{ }$ & & & & \\
\hline 93 & Coelogyne punctulata Lindl. & $\sqrt{ }$ & $\sqrt{ }$ & & & & \\
\hline 94 & Coelogyne stricta (D.Don) Schltr. & $\sqrt{ }$ & $\sqrt{ }$ & & & & \\
\hline 95 & Coelogyne suaveolens (Lindl.) Hook.f. & $\sqrt{ }$ & & & & & \\
\hline 96 & Coelogyne viscosa Rchb.f. & $\sqrt{ }$ & & & $\sqrt{ }$ & & \\
\hline 97 & Conchidium muscicola (Lindl.) Rauschert & & $\sqrt{ }$ & & & & \\
\hline 98 & Cottonia peduncularis (Lindl.) Rchb.f. & & & & & & \\
\hline 99 & Cremastra appendiculata (D.Don) Makino & & $\sqrt{ }$ & $\sqrt{ }$ & & & \\
\hline 100 & Crepidium acuminatum (D.Don) Szlach. & $\sqrt{ }$ & $\sqrt{ }$ & $\sqrt{ }$ & & & \\
\hline 101 & $\begin{array}{l}\text { Crepidium bidentiferum (J.J.Sm.) Marg. and } \\
\text { Szlach. }\end{array}$ & & & & & & \\
\hline
\end{tabular}


102 Crepidium khasianum (Hook.f.) Szlach.

103 Cryptochilus luteus Lindl.

104 Cryptochilus sanguineus Wall.

105 Cymbidium aloifolium (L) Sw.

106 Cymbidium bicolor Lindl.

107 Cymbidium cochleare Lindl.

108 Cymbidium cyperifolium Wall. ex Lindl.

109 Cymbidium dayanum Rchb.f.

110 Cymbidium devonianum Paxton

111 Cymbidium eburneum Lindl

112 Cymbidium elegans Lindl.

113 Cymbidium ensifolium (L.) Sw.

114 Cymbidium erythraeum Lindl

115 Cymbidium gammieanum King and Pantl.

116 Cymbidium hookerianum Rchb.f.

117 Cymbidium iridioides D.Don

118 Cymbidium lancifolium Hook.

119 Cymbidium lowianum (Rchb.f.) Rchb.f.

120 Cymbidium macrorhizon Lindl.

121 Cymbidium mastersii Griff. ex Lindl.

122 Cymbidium munronianum King and Pantl

123 Cymbidium tigrinum C.S.P.Parish ex Hook.

124 Cymbidium tracyanum L.Castle

125 Dendrobium acinaciforme Roxb.

126 Dendrobium aduncum Lindl.

127 Dendrobium amoenum Wall. ex Lindl.

128 Dendrobium amplum Lindl.

129 Dendrobium anceps Sw.

130 Dendrobium aphyllum (Roxb.) C.E.C.Fisch.

131 Dendrobium aqueum Lindl.

132 Dendrobium bellatulum Rolfe

133 Dendrobium bensoniae Rchb.f.

134 Dendrobium bicameratum Lindl.

135 Dendrobium capillipes Rchb.f.

136 Dendrobium chrysanthum Wall. ex Lindl.

137 Dendrobium chryseum Rolfe

138 Dendrobium chrysotoxum Lindl.

139 Dendrobium crepidatum Lindl. and Paxton

140 Dendrobium crumenatum Sw.

141 Dendrobium cumulatum Lindl.

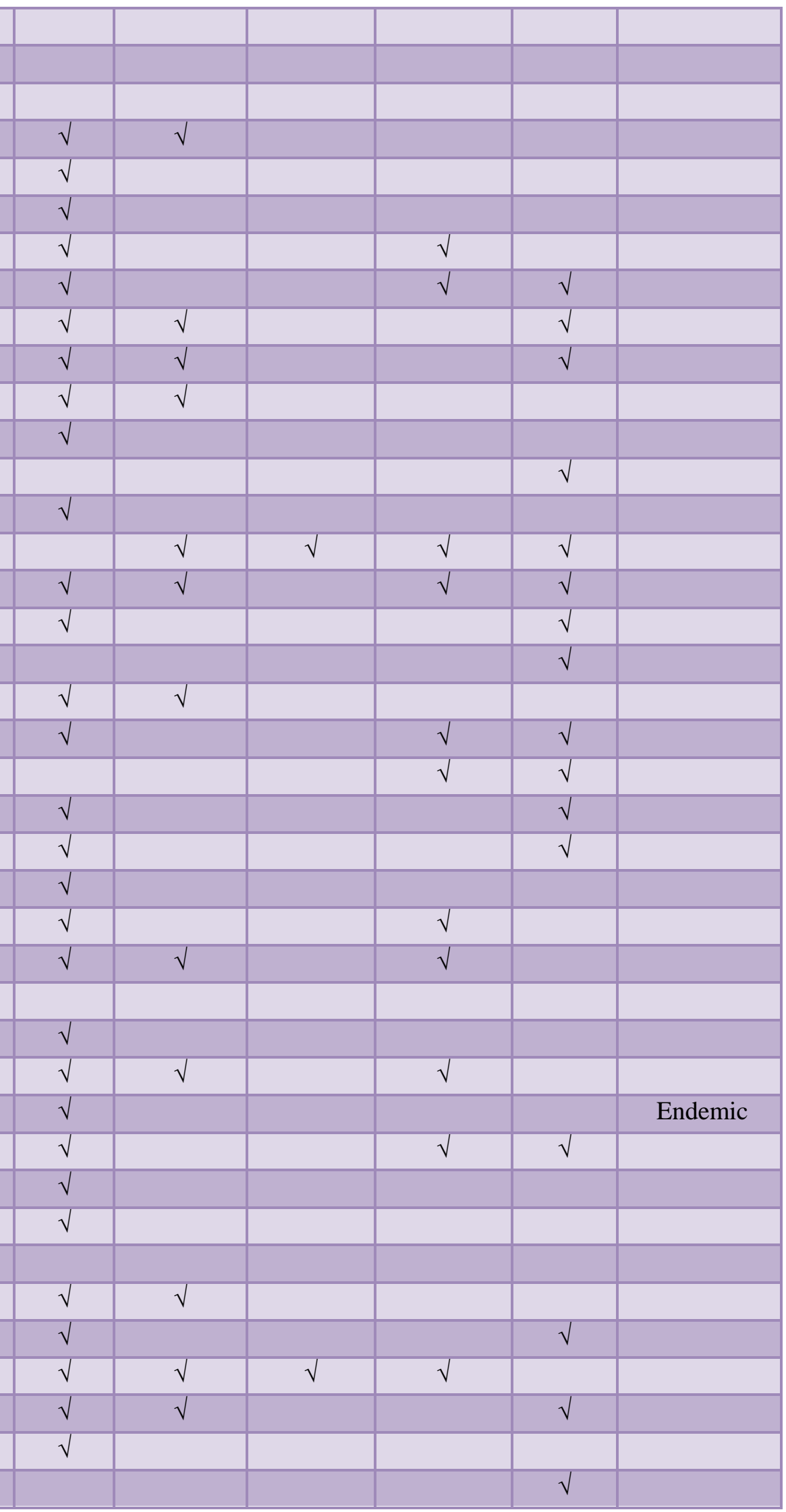




\begin{tabular}{|c|c|c|c|c|c|c|c|}
\hline 142 & Dendrobium densiflorum Lindl. & $\checkmark$ & $\checkmark$ & & $\sqrt{ }$ & & \\
\hline 143 & Dendrobium denudans D.Don & $\sqrt{ }$ & $\sqrt{ }$ & & $\sqrt{ }$ & & \\
\hline 144 & Dendrobium devonianum Paxton & $\sqrt{ }$ & $\sqrt{ }$ & & & & \\
\hline 145 & Dendrobium draconis Rchb.f. & $\sqrt{ }$ & & & $\sqrt{ }$ & $\sqrt{ }$ & \\
\hline 146 & Dendrobium eriiflorum Griff. & $\sqrt{ }$ & $\sqrt{ }$ & & $\sqrt{ }$ & & \\
\hline 147 & Dendrobium falconeri Hook. & $\checkmark$ & $\checkmark$ & & $\sqrt{ }$ & $\checkmark$ & \\
\hline 148 & Dendrobium farmeri Paxton & $\sqrt{ }$ & & & & & \\
\hline 149 & Dendrobium fimbriatum Hook. & $\sqrt{ }$ & $\sqrt{ }$ & & $\sqrt{ }$ & & \\
\hline 150 & Dendrobium fimbriatum var. oculatum & & & & & $\checkmark$ & \\
\hline 151 & Dendrobium formosum Roxb. ex Lindl. & $\sqrt{ }$ & & & & & \\
\hline 152 & Dendrobium fugax Rchb.f. & $\sqrt{ }$ & $\sqrt{ }$ & & & & \\
\hline 153 & Dendrobium gratiosissimum Rchb.f. & $\sqrt{ }$ & & & & & \\
\hline 154 & Dendrobium herbaceum Lindl. & $\sqrt{ }$ & & & & & Endemic \\
\hline 155 & Dendrobium heterocarpum Wall. ex Lindl. & $\sqrt{ }$ & $\sqrt{ }$ & & $\sqrt{ }$ & & \\
\hline 156 & Dendrobium hookerianum Lindl. & $\sqrt{ }$ & & & $\sqrt{ }$ & $\sqrt{ }$ & \\
\hline 157 & Dendrobium infundibulum Lindl. & $\sqrt{ }$ & & & & & \\
\hline 158 & Dendrobium jenkinsii Wall. ex Lindl. & $\sqrt{ }$ & $\checkmark$ & & & & \\
\hline 159 & Dendrobium kingianum Bidwill ex Lindl. & & & $\sqrt{ }$ & & & \\
\hline 160 & Dendrobium lindleyi Steud. & $\sqrt{ }$ & & & $\sqrt{ }$ & & \\
\hline 161 & Dendrobium lituiflorum Lindl. & $\sqrt{ }$ & & & & & \\
\hline 162 & Dendrobium loddigesii Rolfe & & & & & & \\
\hline 163 & Dendrobium longicornu Lindl. & $\sqrt{ }$ & $\sqrt{ }$ & & $\sqrt{ }$ & & \\
\hline 164 & Dendrobium macraei Lindl. & & $\checkmark$ & & & & \\
\hline 165 & Dendrobium macrostachyum Lindl. & $\sqrt{ }$ & & & $\sqrt{ }$ & & \\
\hline 166 & Dendrobium moniliforme (L.) Sw. & $\checkmark$ & $\checkmark$ & & $\sqrt{ }$ & & \\
\hline 167 & Dendrobium moschatum (Buch.-Ham.) Sw. & $\sqrt{ }$ & $\sqrt{ }$ & & $\sqrt{ }$ & & \\
\hline 168 & Dendrobium nanum Hook.f. & $\sqrt{ }$ & & & & $\sqrt{ }$ & Endemic \\
\hline 169 & Dendrobium nobile Lindl. & $\sqrt{ }$ & $\sqrt{ }$ & $\sqrt{ }$ & $\sqrt{ }$ & $\sqrt{ }$ & \\
\hline 170 & Dendrobium nobile Lindl. (White-albus) & $\sqrt{ }$ & $\checkmark$ & $\sqrt{ }$ & & $\checkmark$ & Variant \\
\hline 171 & $\begin{array}{l}\text { Dendrobium nobile Lindl. (Var. } \\
\text { schroderianum) }\end{array}$ & $\sqrt{ }$ & $\sqrt{ }$ & $\sqrt{ }$ & & $\checkmark$ & Variant \\
\hline 172 & Dendrobium nodosum Dalzell & $\sqrt{ }$ & & & & & \\
\hline 173 & Dendrobium ochreatum Lindl. & $\sqrt{ }$ & & & $\sqrt{ }$ & & \\
\hline 174 & Dendrobium ovatum (L.) Kraenzl. & $\sqrt{ }$ & & & & $\checkmark$ & Endemic \\
\hline 175 & Dendrobium parishii Rchb.f. & $\sqrt{ }$ & & & $\sqrt{ }$ & $\checkmark$ & \\
\hline 176 & Dendrobium pendulum Roxb. & $\sqrt{ }$ & & & & & \\
\hline 177 & Dendrobium plicatile. (Lindl.) & $\sqrt{ }$ & & & $\sqrt{ }$ & & \\
\hline 178 & Dendrobium polyanthum Wall. ex Lindl. & $\sqrt{ }$ & & & $\sqrt{ }$ & & \\
\hline 179 & Dendrobium porphyrochilum Lindl. & $\sqrt{ }$ & & & & & \\
\hline 180 & Dendrobium praecinctum Rchb.f. & $\sqrt{ }$ & & & & $\sqrt{ }$ & \\
\hline
\end{tabular}


181 Dendrobium primulinum Lindl.

182 Dendrobium pulchellum Roxb. ex Lindl.

183 Dendrobium rotundatum (Lindl.) Hook.f.

184 Dendrobium ruckeri Lindl.

185 Dendrobium salaccense (Blume) Lindl.

186 Dendrobium stuposum Lindl.

187 Dendrobium sulcatum Lindl.

188 Dendrobium terminale E.C.Parish and Rchb.f.

189 Dendrobium thyrsiflorum B.S.Williams

190 Dendrobium transparens Wall. ex Lindl.

191 Dendrobium wardianum R.Warner

192 Dendrobium williamsonii Day and Rchb.f.

193 Dienia ophrydis (J.Koenig) Seidenf.

194 Diplocentrum recurvum Lindl.

195 Diplomeris hirsuta (Lindl.) Lindl.

196 Epidendrum ellipticum Graham

197 Epidendrum radicans Pav. ex Lindl.

198 Epidendrum xanthinum Lindl.

199 Epipogium roseum (D.Don) Lindl.

200 Eria clausa King and Pantl.

201 Eria coronaria (Lindl.)Rchb.f.

202 Eria ferruginea Lindl.

203 Eria globulifera Seidenf.

204 Eria javanica (Sw.) Blume

205 Eria lasiopetala (Willd.) Ormerod

206 Eria porteri Seidenf. and A.D.Kerr

207 Eria sutepensis Rolfe ex Downie

208 Eria tomentosa (J.Koenig) Hook.f.

209 Eria vittata Lindl.

210 Esmeralda cathcartii (Lindl.) Rchb.f.

211 Esmeralda clarkei Rchb.f.

212 Eulophia pulchra (Thouars) Lindl

213 Gastrochilus acutifolius (Lindl.) Kuntze

214 Gastrochilus calceolaris (Buch.-Ham. ex Sm.) D.Don

215 Gastrochilus dasypogon (Sm.) Kuntze

216 Gastrochilus distichus (Lindl.) Kuntze

217 Gastrochilus inconspicuus (Hook.f.) Kuntze

218 Gastrochilus pseudodistichus (King and Pantl.) Schltr.

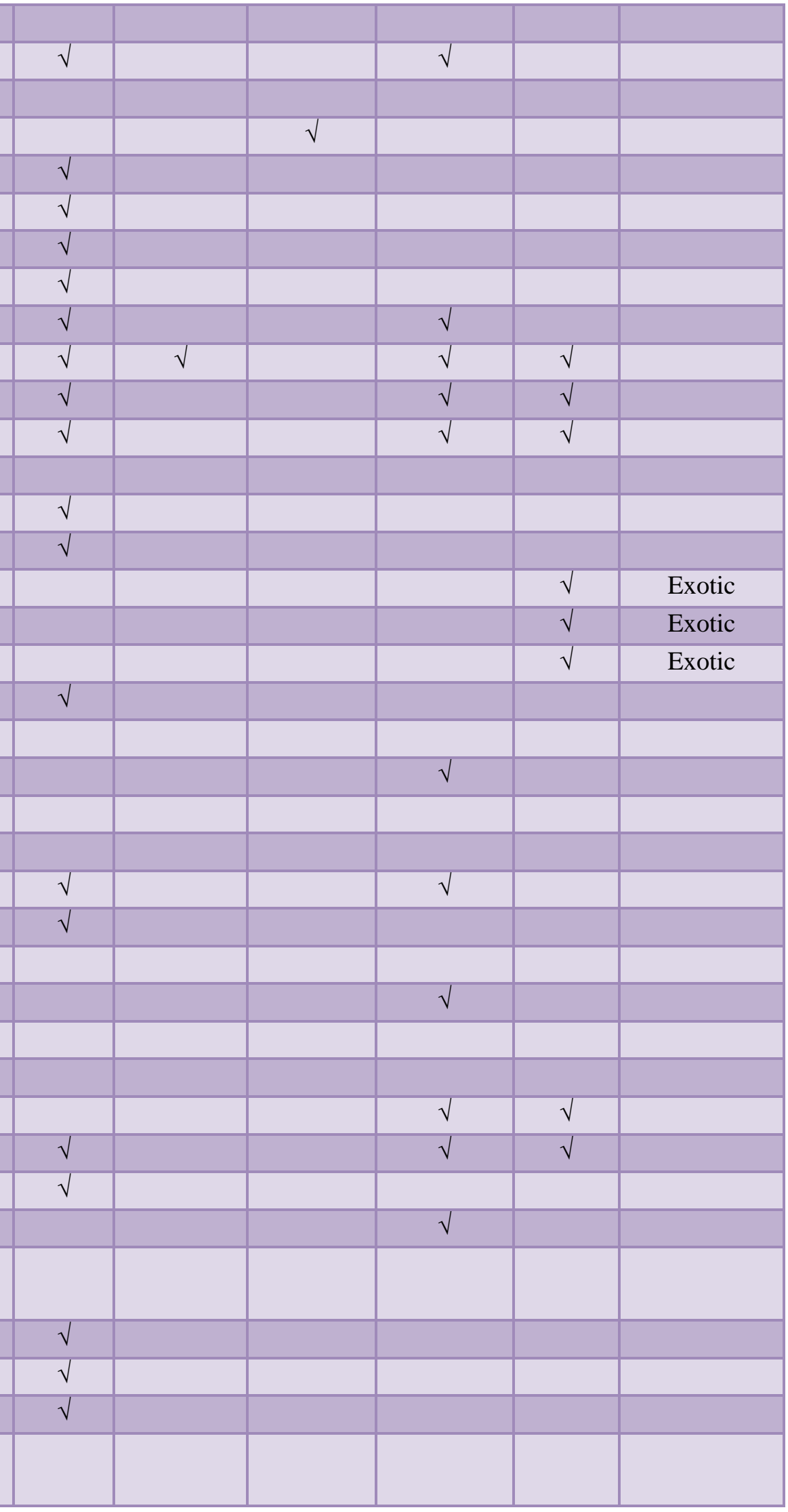


219 Geodorum densiflorum (Lam.) Schltr.

210 Goodyera foliosa (Lindl.) Benth. ex C.B.Clarke

211 Goodyera hispida Lindl.

212 Goodyera hemsleyana King and Pantl.

213 Goodyera procera (Ker Gawl.) Hook.

214 Goodyera schlechtendaliana Rchb.f.

215 Guarianthe bowringiana (O'Brien) Dressler and W.E.Higgins

216 Habenaria arietina Hook.f.

217 Habenaria furcifera Lindl.

218 Herminium lanceum (Thunb. ex Sw.) Vuijk

219 Herminium mackinnonii Duthei

220 Herpysma longicaulis Lindl.

221 Holcoglossum amesianum (Rchb.f.) Christenson

222 Hygrochilus parishii (Veitch and Rchb.f.) Pfitzer

223 Liparis bistriata E.C.Parish and Rchb.f.

224 Liparis bootanensis Griff.

225 Liparis cespitosa (Lam.) Lindl.

226 Liparis cordifolia Hook.f.

227 Liparis deflexa Hook.f.

228 Liparis elliptica Wight

229 Liparis odorata (Willd.) Lindl.

230 Liparis latifolia Lindl.

231 Liparis plantaginea Lindl.

232 Liparis resupinata Ridl.

233 Liparis viridiflora (Blume) Lindl.

234 Luisia brachystachys (Lindl.) Blume

235 Luisia filiformis Hook.f.

236 Luisia trichorrhiza (Hook.) Blume

237 Luisia tristis (G.Forst.) Hook.f.

238 Lycaste cruenta (Lindl.) Lindl.

239 Malaxis rheedii B. Heyne ex Wallace

240 Masdevallia ignea Rchb.f.

241 Micropera mannii (Hook.f.) Tang and F.T.Wang

242 Micropera obtusa (Lindl.) Tang and F.T.Wang

243 Micropera pallida (Roxb.) Lindl.

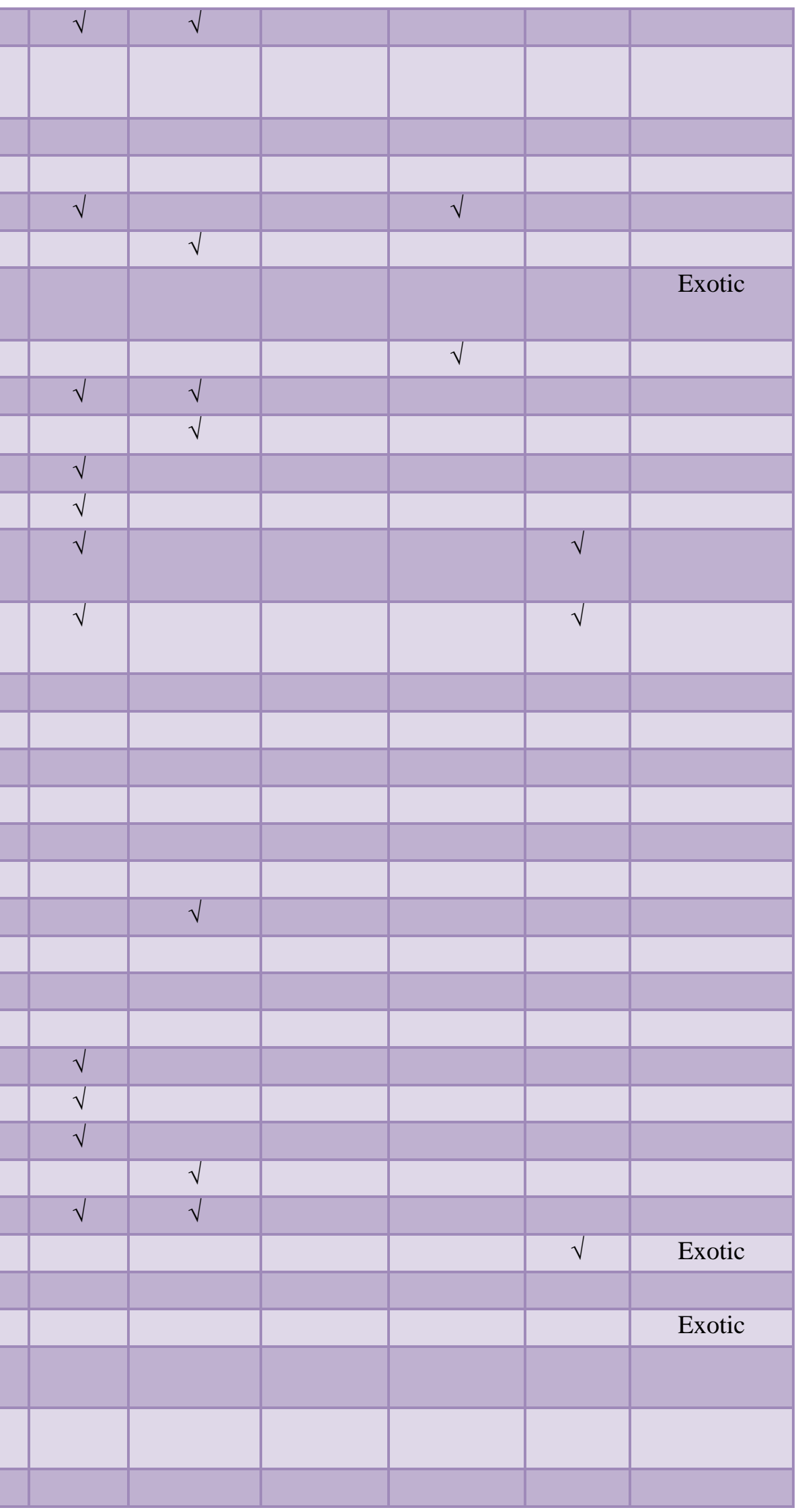


244 Mormolyca rufescens (Lindl.) M.A.Blanco

245 Mycaranthes floribunda (D.Don) S.C.Chen and J.J.Wood

246 Neogyna gardneriana (Lindl.) Rchb.f.

247 Nervilia macroglossa (Hook.f.) Schltr.

248 Oberonia acaulis Griff.

249 Oberonia cylindrica Lindl.

250 Oberonia emarginata King and Pantl.

251 Oberonia obcordata Lindl.

252 Oberonia pachyrachis Rchb.f. ex Hook.f.

253 Oberonia prainiana King and Pantl.

254 Odontochilus lanceolatus (Lindl.) Blume

255 Ornithochilus difformis (Wall. ex Lindl.) Schltr.

256 Otochilus albus Lindl.

257 Otochilus fuscus Lindl.

258 Otochilus porrectus Lindl.

259 Panisea demissa (D.Don) Pfitzer

260 Panisea uniflora (Lindl.) Lindl.

261 Paphiopedilum fairrieanum (Lindl.) Stein

262 Paphiopedilum hirsutissimum (Lindl. ex Hook.) Stein

263 Paphiopedilum insigne (Wall. ex Lindl.) Pfitzer

264 Paphiopedilum spicerianum (Rchb.f.) Pfitzer

265 Paphiopedilum venustum (Wall. ex Sims) Pfitzer

266 Paphiopedilum villosum (Lindl.) Stein

267 Paphiopedilum villosum var. boxallii (Rchb.f.) Pfitzer

268 Papilionanthe subulata (Willd.) Garay

269 Papilionanthe teres (Roxb.) Schltr.

270 Papilionanthe uniflora (Lindl.) Garay

271 Papilionanthe vandarum (Rchb.f.) Garay

272 Pelatantheria insectifera (Rchb.f.) Ridl.

273 Phaius flavus (Blume) Lindl.

274 Phaius mishmensis (Lindl. and Paxton) Rchb.f.

275 Phaius tankervilleae (Banks) Blume

276 Phalaenopsis deliciosa Rchb.f.

277 Phalaenopsis lobbii (Rchb.f.) H.R.Sweet

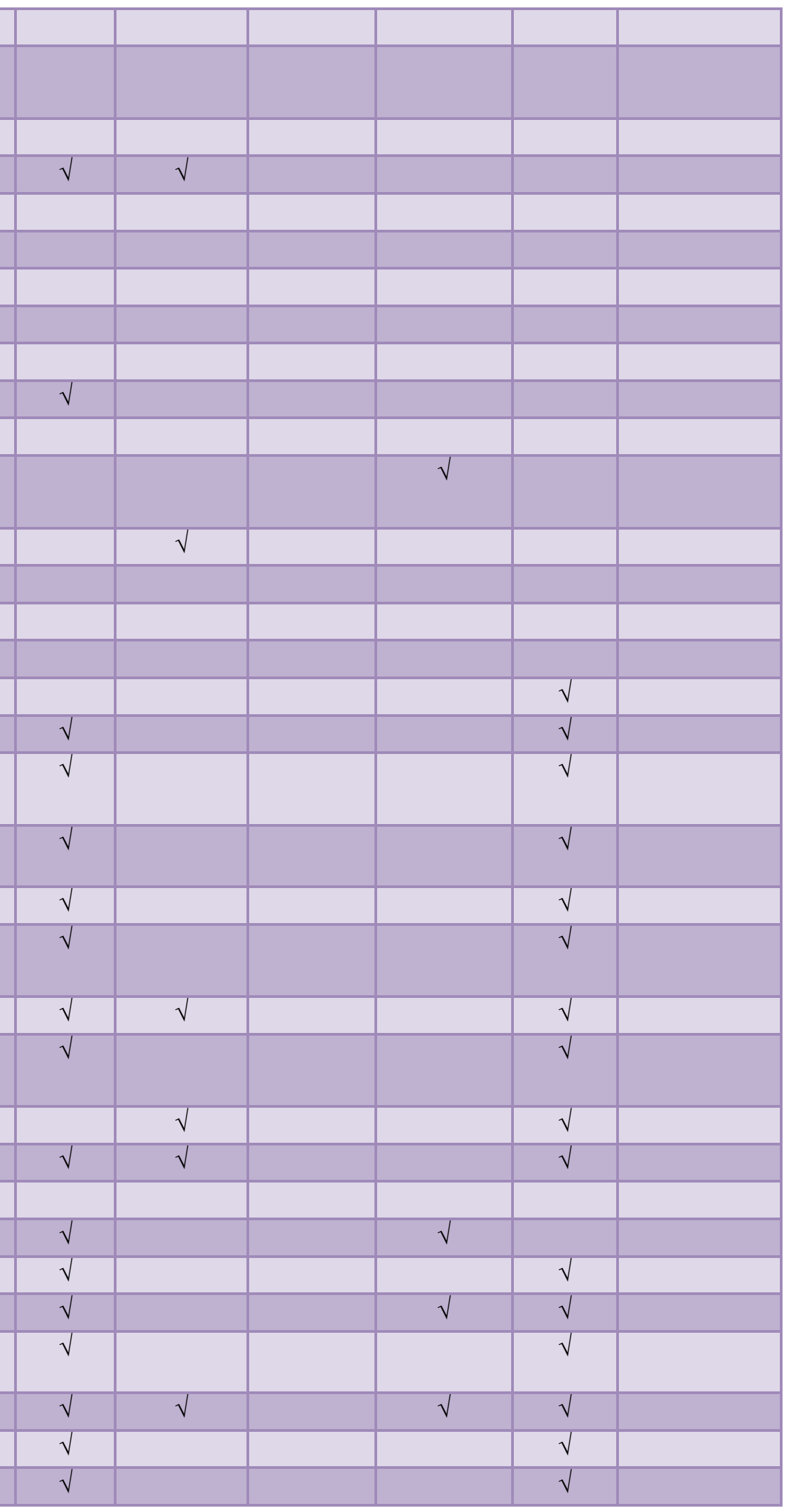


278 Phalaenopsis mannii Rchb.f.

279 Phalaenopsis taenialis (Lindl.) Christenson and Pradhan

280 Pholidota articulata Lindl.

281 Pholidota imbricata Lindl.

282 Pholidota pallida Lindl.

283 Pholidota protracta Hook.f.

284 Pholidota recurva Lindl.

285 Phreatia elegans Lindl.

286 Phreatia laxiflora (Blume) Lindl.

287 Pinalia acervata (Lindl. ) Kuntze

288 Pinalia amica (Rchb.f.) Kuntze

289 Pinalia bipunctata (Lindl.) Kuntze

290 Pinalia bractescens(Lindl.) Kuntze

291 Pinalia excavata (Lindl.) Kuntze

292 Pinalia graminifolia (Lindl.) Kuntze

293 Pinalia pumila (Lindl.) Kuntze

294 Pinalia spicata (D.Don) S.C.Chen and J.J.Wood

295 Platanthera clavigera Lindl.

296 Pleione hookeriana (Lindl.) Rollisson

297 Pleione humilis (Sm.) D.Don

298 Pleione maculata (Lindl.) Lindl. and Paxton

299 Pleione praecox (Sm.) D.Don

300 Podochilus cultratus Lindl.

301 Podochilus malabaricus Wight

302 Polystachya concreta (Jacq.) Garay and H.R.Sweet

303 Pomatocalpa armigerum (King and Pantl.) Tang and F.T.Wang

304 Pteroceras teres (Blume) Holttum

305 Renanthera imschootiana Rolfe

306 Rhynchostylis retusa (L.) Blume

307 Satyrium nepalense D.Don

308 Schoenorchis gemmata (Lindl.) J.J.Sm.

309 Schoenorchis smeeana (Rchb.f.)

310 Sirhookera latifolia (Wight) Kuntze

311 Smitinandia micrantha (Lindl.) Holttum

312 Spiranthes sinensis (Pers.) Ames

313 Staurochilus ramosus (Lindl.) Seidenf.

\begin{tabular}{|c|c|c|c|c|c|}
\hline$\sqrt{ }$ & & & $\checkmark$ & & \\
\hline$\sqrt{ }$ & & & & & \\
\hline$\sqrt{ }$ & $\sqrt{ }$ & & $\sqrt{ }$ & & \\
\hline$\sqrt{ }$ & $\sqrt{ }$ & & $\sqrt{ }$ & & \\
\hline$\sqrt{ }$ & & & & & \\
\hline & & & & & \\
\hline & & & & & \\
\hline & & & & & \\
\hline & & & & & \\
\hline & & & $\sqrt{ }$ & & \\
\hline & & & & & \\
\hline & & & & & \\
\hline & & & & & \\
\hline & & & & & \\
\hline & & & & & \\
\hline$\sqrt{ }$ & & & & & \\
\hline$\sqrt{ }$ & $\sqrt{ }$ & & & & \\
\hline & & & & $\sqrt{ }$ & \\
\hline$\sqrt{ }$ & & & & $\sqrt{ }$ & \\
\hline$\sqrt{ }$ & $\sqrt{ }$ & & $\sqrt{ }$ & $\sqrt{ }$ & \\
\hline$\sqrt{ }$ & $\sqrt{ }$ & & $\sqrt{ }$ & $\sqrt{ }$ & \\
\hline$\sqrt{ }$ & $\sqrt{ }$ & & $\sqrt{ }$ & $\sqrt{ }$ & \\
\hline & & & & & \\
\hline & & & & & \\
\hline$\sqrt{ }$ & & & & & \\
\hline & & & & $\sqrt{ }$ & \\
\hline & & & & $\sqrt{ }$ & \\
\hline$\sqrt{ }$ & $\sqrt{ }$ & & $\sqrt{ }$ & $\sqrt{ }$ & \\
\hline & $\sqrt{ }$ & $\sqrt{ }$ & $\sqrt{ }$ & & \\
\hline$\sqrt{ }$ & & & & & \\
\hline & & & & & Endemic \\
\hline & & & & & \\
\hline$\sqrt{ }$ & $\sqrt{ }$ & & & & \\
\hline$\sqrt{ }$ & & & & & \\
\hline$\sqrt{ }$ & & & & & \\
\hline
\end{tabular}




\begin{tabular}{|c|c|c|c|c|c|c|c|}
\hline 314 & Sunipia bicolorLindl. & $\sqrt{ }$ & & & & & \\
\hline 315 & Sunipia cirrhata (Lindl.) P. F. Hunt & $\sqrt{ }$ & & & & & \\
\hline 316 & Sunipia scariosa Lindl. & & & & & & \\
\hline 317 & $\begin{array}{l}\text { Taeniophyllum retrospiculatum (King and } \\
\text { Pantl.) King and Pantl. }\end{array}$ & & & & & & \\
\hline 318 & Tainia minor Hook.f. & & & & & & \\
\hline 319 & Thelasis longifolia Hook.f. & $\sqrt{ }$ & & & & & \\
\hline 320 & Thelasis pygmaea (Griff.) Lindl. & & & & & & \\
\hline 321 & $\begin{array}{l}\text { Thrixspermum musciflorum A.S.Rao and } \\
\text { J.Joseph }\end{array}$ & $\sqrt{ }$ & & & & & Endemic \\
\hline 322 & Thunia alba (Lindl.) Rchb.f. & $\sqrt{ }$ & $\sqrt{ }$ & & & $\sqrt{ }$ & \\
\hline 323 & $\begin{array}{l}\text { Thunia alba var. bracteata (Roxb.) N. Pearce } \\
\text { and P.J. Cribb }\end{array}$ & & & & & $\sqrt{ }$ & \\
\hline 324 & Thunia alba var. marshalliana (Rchb.f.) & & & & & & \\
\hline 325 & Uncifera obtusifolia Lindl. & & $\sqrt{ }$ & & & & \\
\hline 326 & Vanda alpina (Lindl.) Lindl. & $\sqrt{ }$ & & & $\sqrt{ }$ & $\sqrt{ }$ & \\
\hline 327 & Vanda coerulea Griff. ex Lindl. & & $\sqrt{ }$ & & & $\sqrt{ }$ & \\
\hline 328 & Vanda cristata Wall. ex Lindl. & & $\sqrt{ }$ & & & $\sqrt{ }$ & \\
\hline 329 & Vanda griffithii Lindl. & & & & & $\sqrt{ }$ & \\
\hline 340 & Vanda motesiana Choltco & & & & & & \\
\hline 341 & Vanda pumila Hook.f. & & & & & $\sqrt{ }$ & \\
\hline 342 & Vanda tessellata (Roxb.) Hook. ex G.Don & $\sqrt{ }$ & $\sqrt{ }$ & $\sqrt{ }$ & & $\sqrt{ }$ & \\
\hline 343 & Vanda testacea (Lindl.) Rchb.f. & $\sqrt{ }$ & $\sqrt{ }$ & & & $\sqrt{ }$ & \\
\hline 344 & Vanda thwaitesii Hook.f. & $\sqrt{ }$ & & & & & \\
\hline 345 & Vanda wightii Rchb.f. & $\sqrt{ }$ & & & & & \\
\hline 346 & Vandopsis undulata (Lindl.) J.J.Sm. & $\sqrt{ }$ & & & $\sqrt{ }$ & & \\
\hline 348 & Vanillaaphylla Blume & & & & & $\sqrt{ }$ & \\
\hline 348 & Zeuxine affinis (Lindl.) Benth. ex Hook.f. & & & & & & \\
\hline 349 & Zeuxine flava (Wall. ex Lindl.) Trimen & & & & & & \\
\hline 350 & Zeuxine goodyeroides Lindl. & & & & & & \\
\hline \multirow[t]{2}{*}{351} & Zygopetalum maculatum (Kunth) Garay & & & & $\sqrt{ }$ & $\sqrt{ }$ & Exotic \\
\hline & Total & 205 & 87 & 11 & 77 & 90 & \\
\hline
\end{tabular}


Fig.1 Orchid germplasm collecting states across the country

\section{States where Exploration Programme was Conducted from 1998-2016}

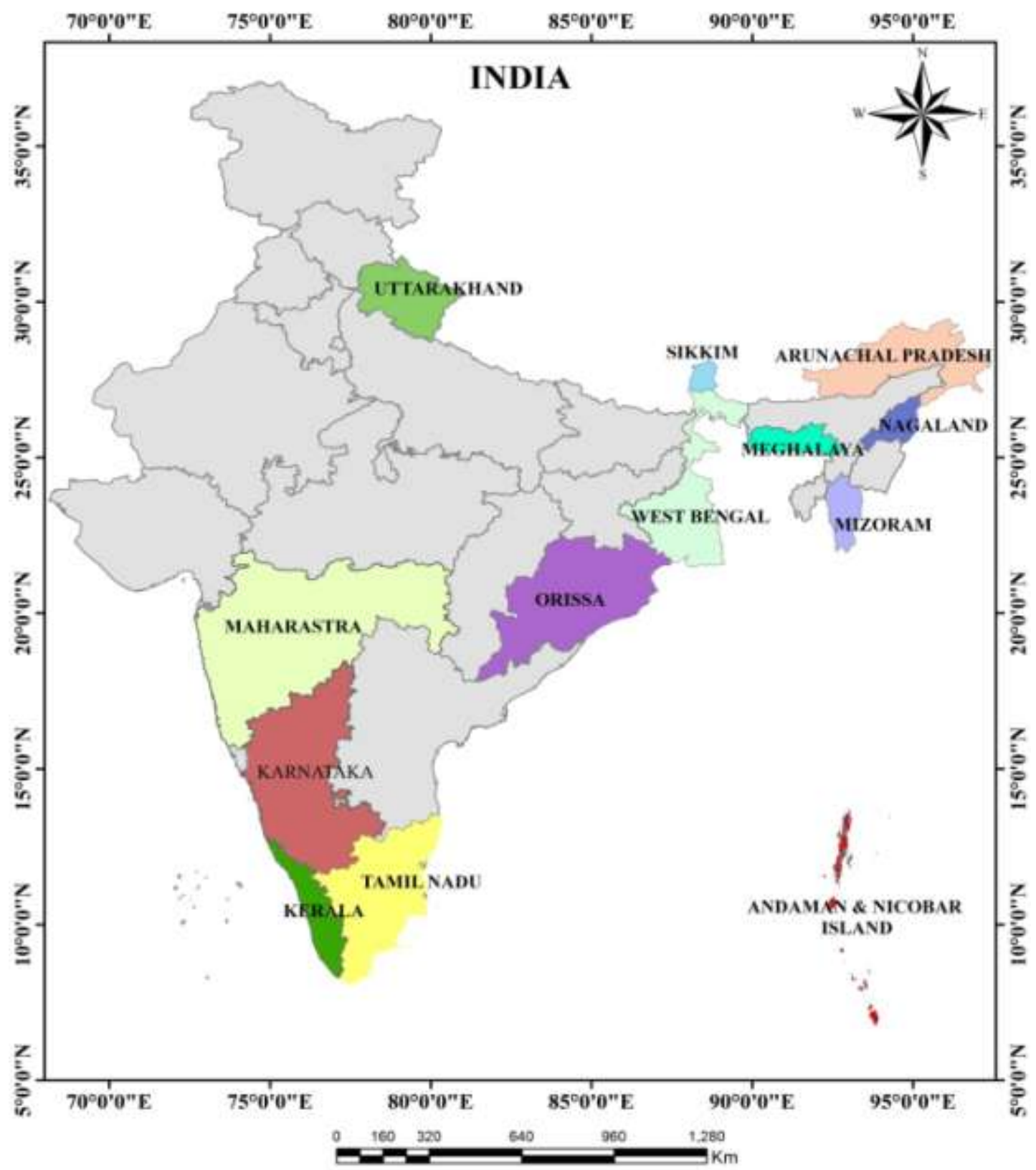


Fig.2 List of rare species which are conserved at ICAR-NRCO

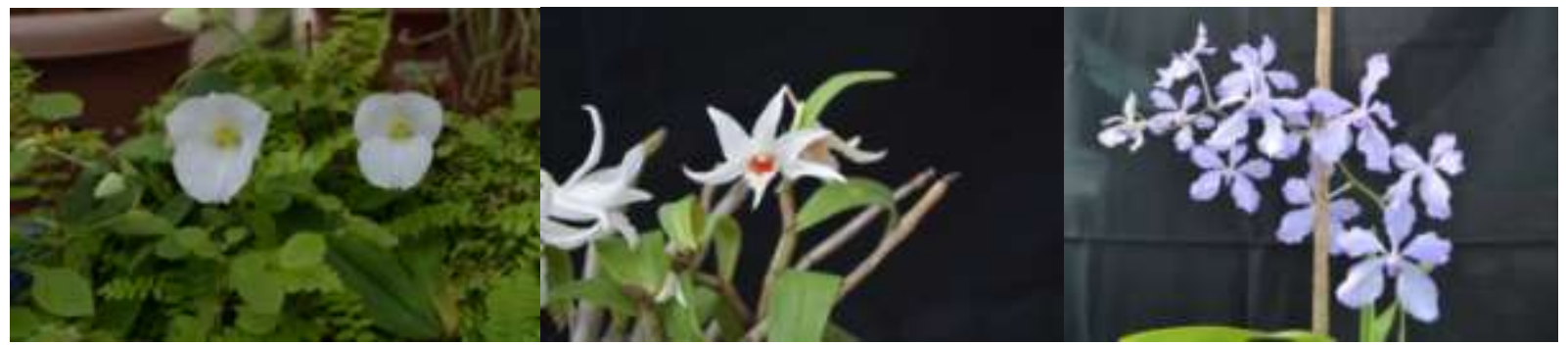

Diplomeris hirsuta (Lindl.)Lindl.Dendrobium draconis Rchb.f.Vanda coerulea

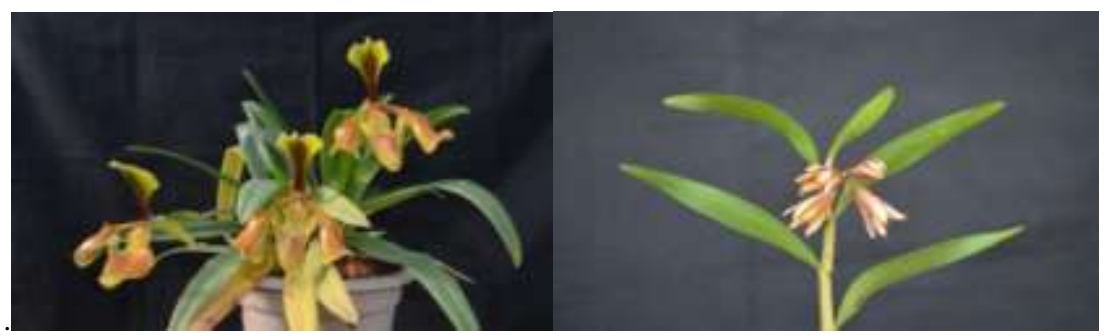

Griff.exLindl

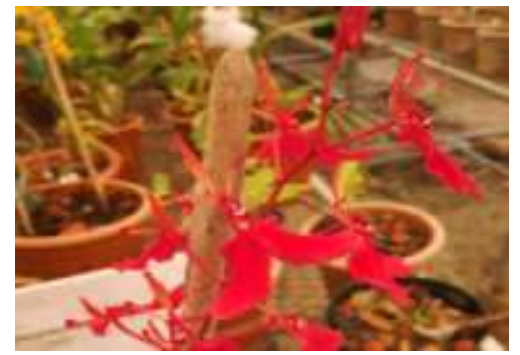

Paphiopedilum villosum (Lindl.)SteinDendrobium praecinctum Rchb.f.Renanthera imschootiana Rolfe

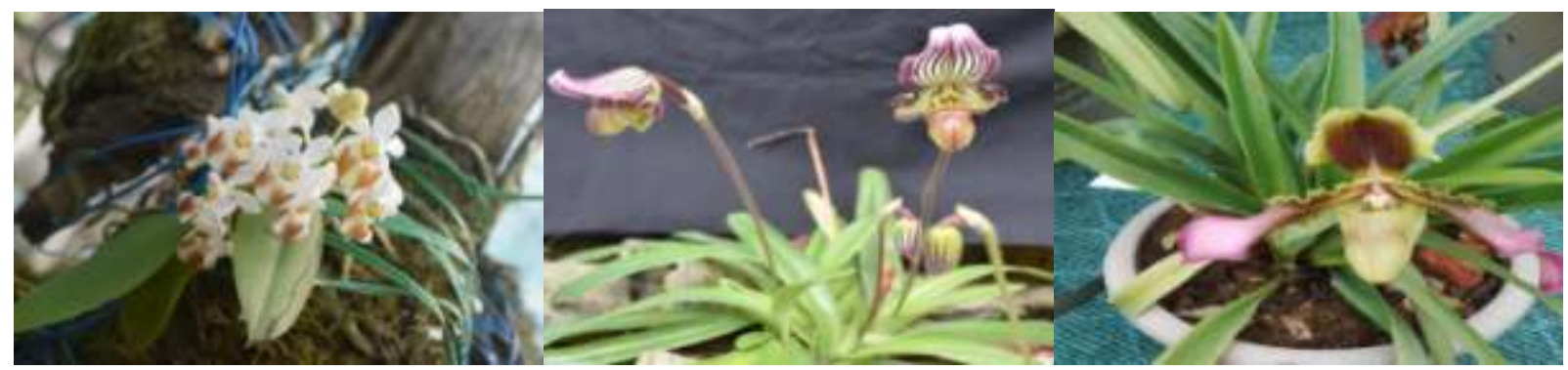

Phalaenopsis lobbii (Rchb.f.) SweetPaphiopedilum fairrieanum (Lindl.)SteinPaphiopedilum hirsutissimum (Lindl. ex Hook.) Stein

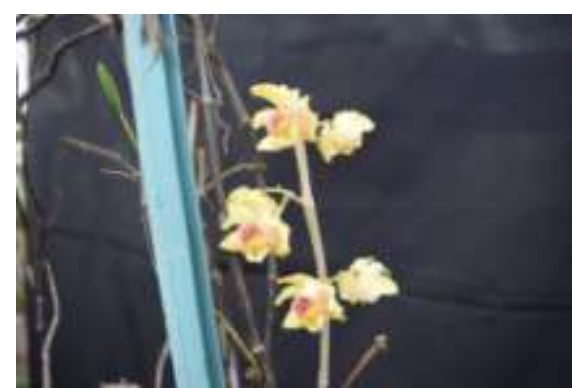

Dendrobium ruckeri Lindl.

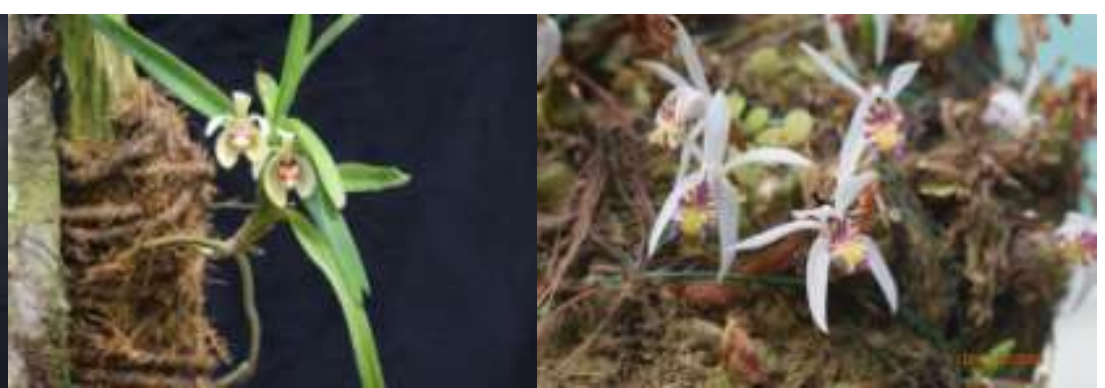

Vanda pumila Hook.f.Pleione maculata (Lindl.)Lindl.\& Paxton 
Fig.3 List of important medicinal orchids

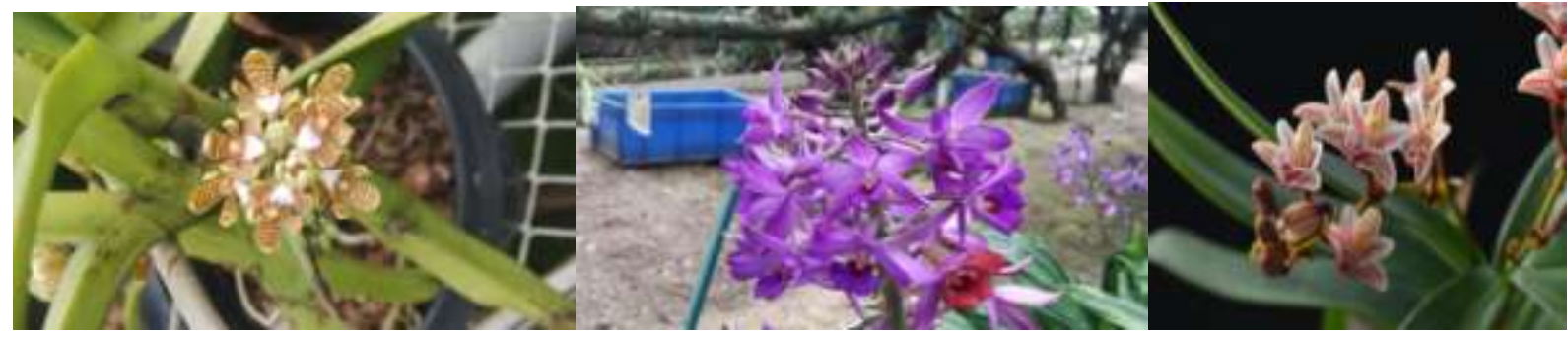

Acampe rigida (Buch.-Ham. ex Sm.)P.F.Hunt Calanthe sylvatica (Thouars) Lindl. Callostylis bambusifolia

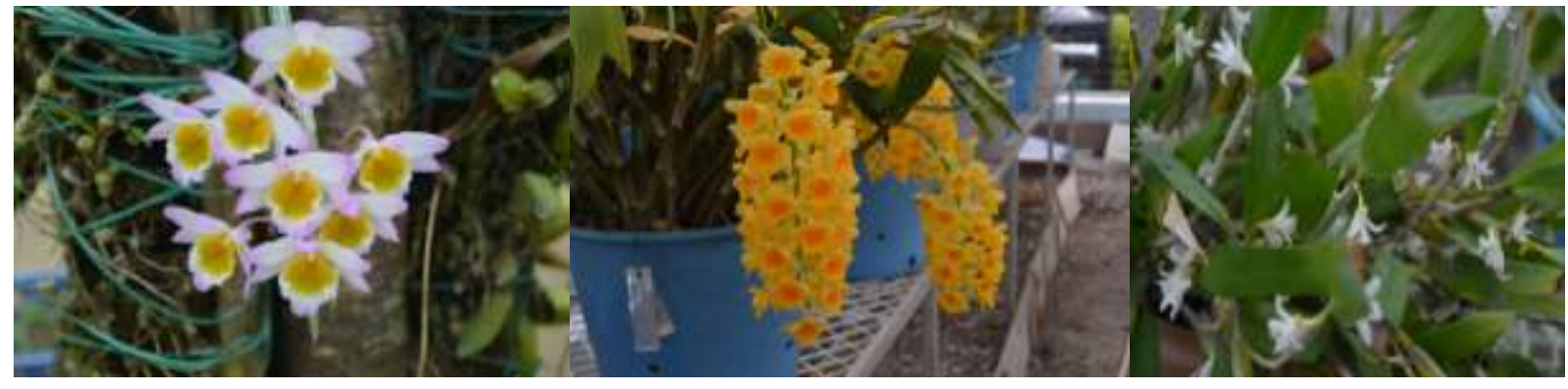

Dendrobium crepidatum Lindl.\& Paxton

Dendrobium densiflorum Lindl.

Dendrobium fugax Rchb.f.

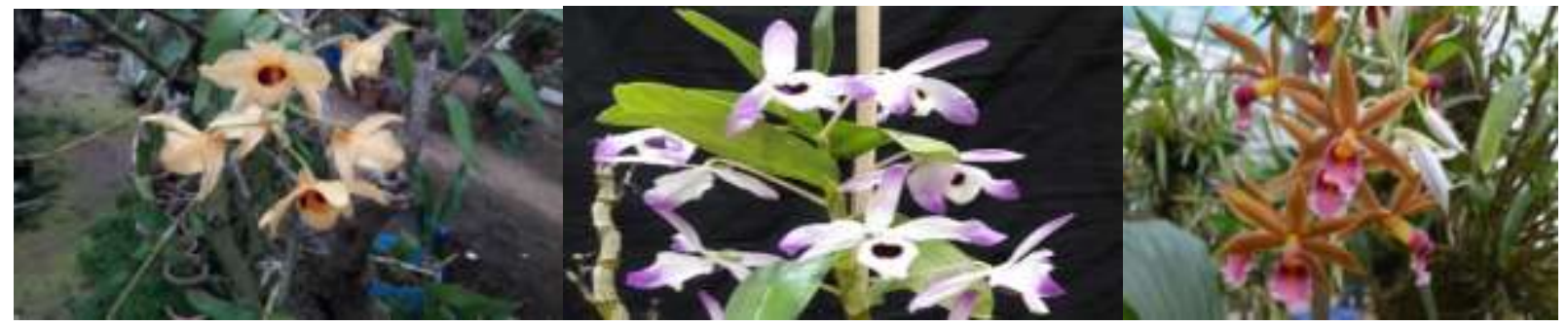

Dendrobium moschatum (Buch.-Ham.)Sw.Dendrobium nobile Lindl.

Phaius tankervilleae Blume

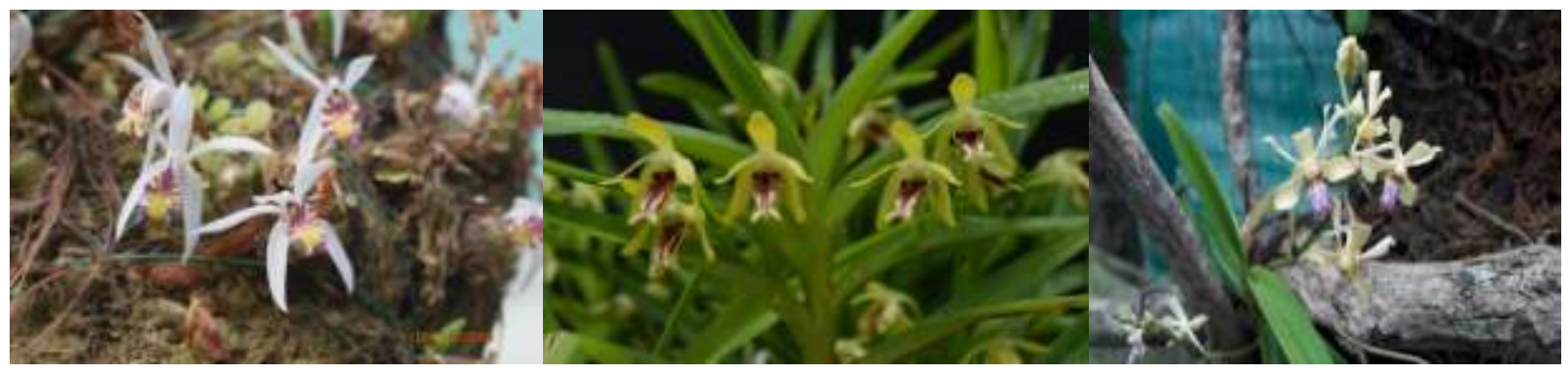

Pleione maculata (Lindl.)Lindl.\& PaxtonVanda cristata Lindl.Vanda testacea (Lindl.)Rchb.f. 
Fig.4 List of important fragrant orchids

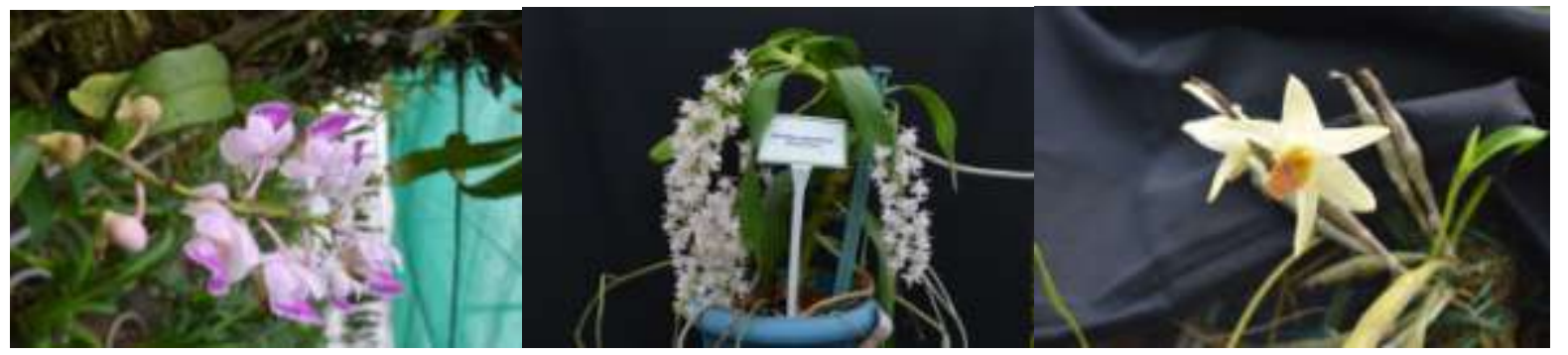

Aerides crispa Lindl

Aerides odoratum Reinw.ex Blume

Dendrobium heterocarpum Wall.ex Lindl.

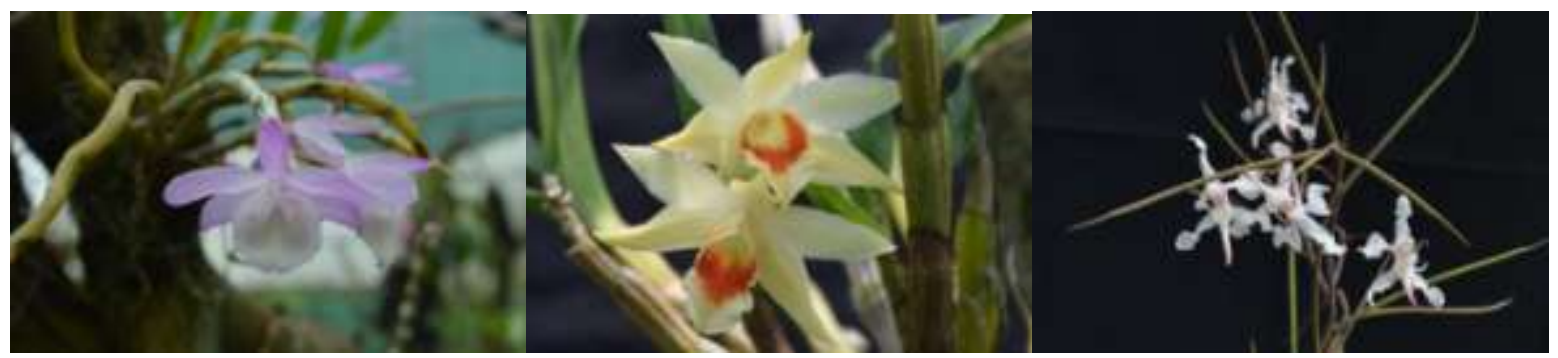

Dendrobium primulinum Lindl.Dendrobium williamsonii Day and Rchb.f.Papilionanthe uniflora (Lindl.) Garay

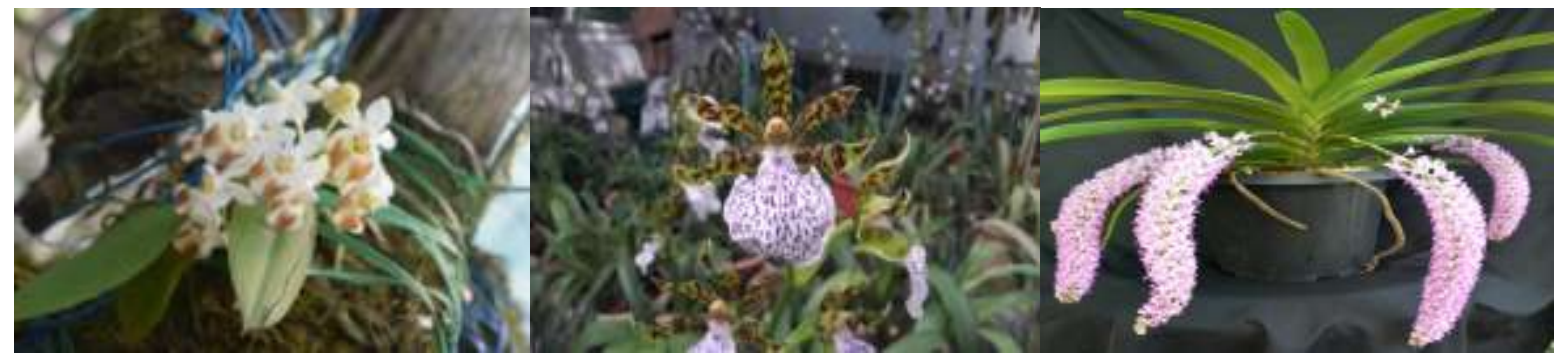

Phalaenopsis lobbii (Rchb.f.) SweetZygopetalum maculatum (Kunth) GarayRhynchostylis retusa (L) Blume

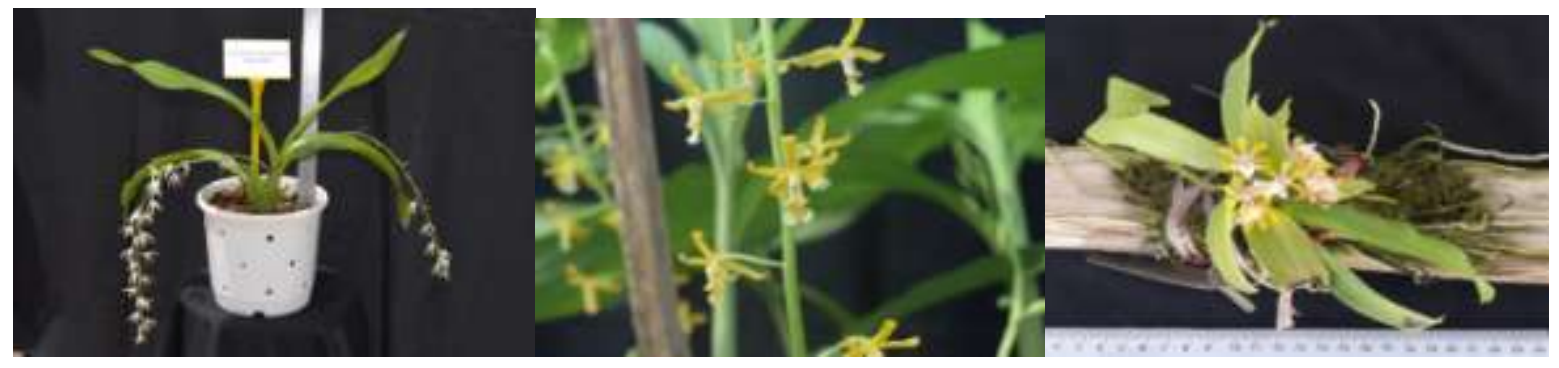

Eria javanica (Sw.) BlumeCephalamtheropsis obcordataGastrochilus dasypogon (Sm.) Kuntze 
Fig.5 Important conservation methods (Field gene bank and DNA bank)

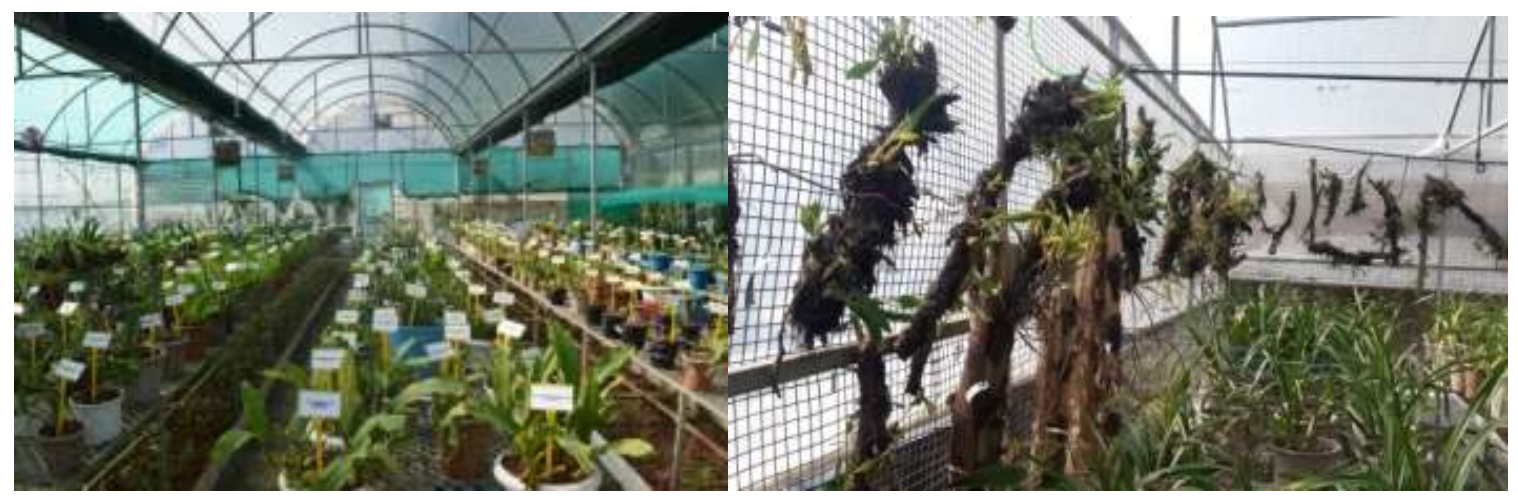

National Active Germplasm Site (NAGS) - Germplasm Polyhouse

Vertically arrangement of germplasm

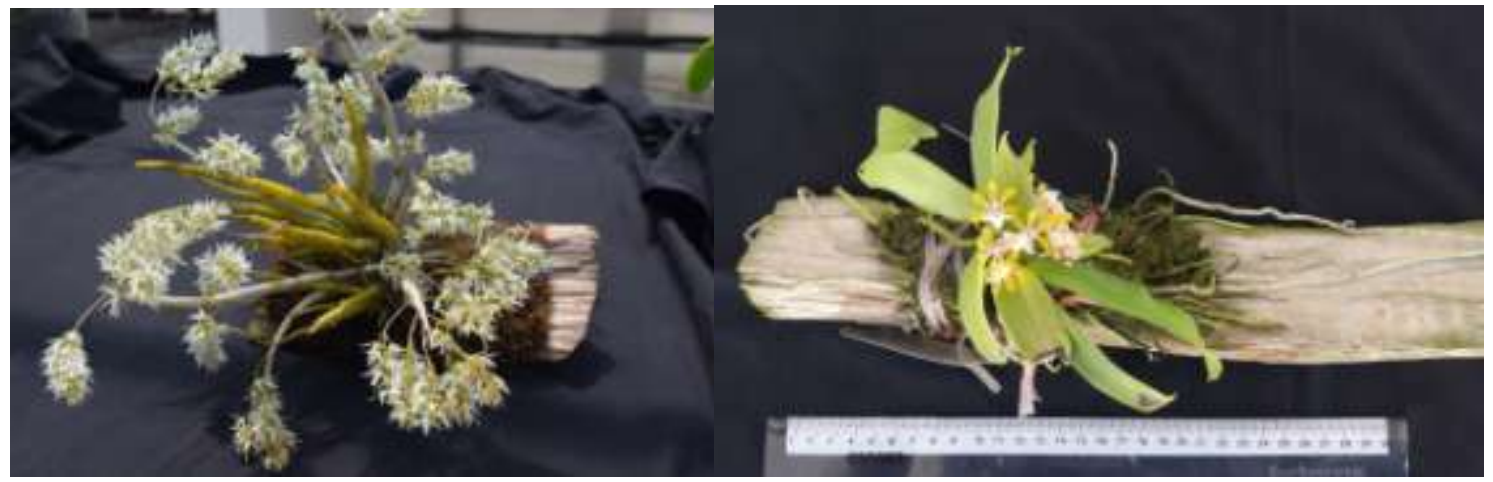

Dendrobium eryflorum and Gastrochils dasypogan on wooden logs

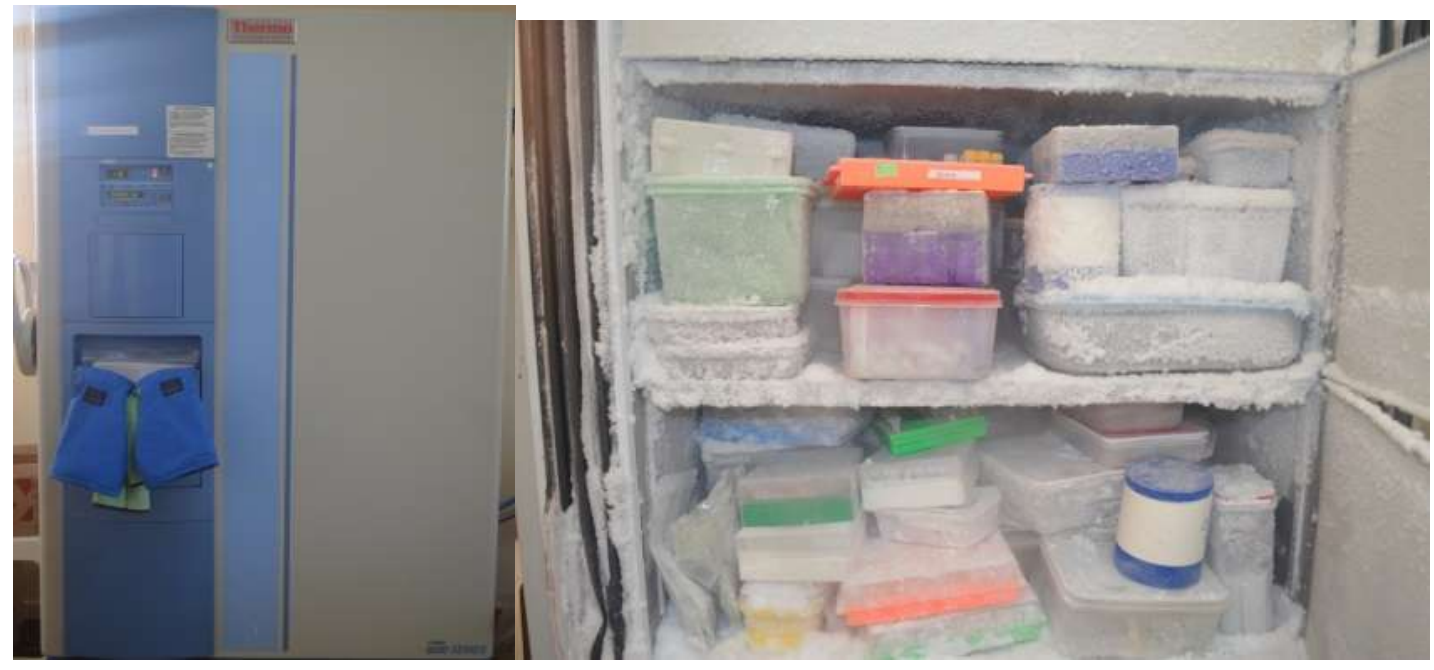

DNA Repository

DNA are stored under $-80^{\circ} \mathrm{C}$ 
In Ayurveda, a rejuvenating herbal formulation 'Astavarga' (Chief component of ayurvedic tonic 'Chyavanprash') is derived from a group of 8 herbs, 4 of them are orchids namely Jivak (Malaxis muscifera), Rishbhaka (Malaxis acuminata), Riddhi (H. intermedia) and Vriddhi (H. edgeworthii) (Uniyal, 1975).

\section{Orchids as food}

Orchid's importance comes into account in traditional food as a side dishes or a supplement in many parts of the world. There are many wild orchid species which are being used a food by the tribal people of North East India (Duggal, 1972). Many tribes of the Nagaland state used leaves of Cymbidium species as food. The new shoots of Cymbidiums are used with cereals to make sauce, the pseudobulbs of their orchids in combination of common vegetables such as potato, tapioca etc. (Medhi and Chakrabarti, 2009). Pseudobulb, root and rhizome of many orchid species, are reported to use as food viz. Habenaria acuminata, H. susannae, Orchis latifolia, Pholidata articulata, Satyrium species are used as foods which play an important role in the nutrition of the people of this Nagaland region (Deb 2013).

\section{Endemism}

The endemism in the flora of a country or geographical region provides an important insight into the biogeography of that region and also to the centers of diversity and adaptive evolution of the floristic components of that region (Nayar 1996). In India, the Himalayan region has a high degree of endemism making it the richest endemic centre. There are about 307 orchid species are belongs to endemic category (Singh 2019). At present we collected rare and endemic orchid species such as Diplomeris hirsuta, Lecanorchis sikkimensis, Phalaenopsis lobbii, Renanthera imschootiana etc. These species are very specific to habitat and sensitive to micro environment.

A number of species are rare and threatened throughout the India, owing to habitat degradation and fragmentation as a result of various anthropogenic influences such as land development activities, building of dams, constructions of roads, commercial exploitation of the species, overgrazing and frequent forest fires. Some orchid species require unique habitat and microhabitats so they are confined to particular elevations and forest types. Some are naturally rare; others are so because of geographic distribution, narrow habitat requirements, and low-density populations.

\section{Conservation}

Conservation of orchids through ex-situ and in-situ methods, in the ex-situ mode, the orchid species are collected from the native location and conserved in the by tying the plants on wooden logs. While in the in-situ approach, the plants are attached with the help of mosses and knotted them tightly with the help of coir ropes. Due to the nonendospermic seeds, the species are normally conserved in the form of field genebanks. Second method is conserving DNA in the form of leaf samples at $-80^{\circ} \mathrm{C}$ in deep freezer.

DNA Bank and NCBI Deposits: Genomic DNA have been isolated from 260 species and stored. 65 DNA barcode sequences (using ITS, matK, rbcL, trnH-psbA primers) were submitted to NCBI.

DNA repository of orchids: The DNA of native orchids is being preserved under $-80^{0} \mathrm{C}$. Nearly 250 species samples are preserved carefully.

North Eastern Himalaya is opulent in orchid resources and indigenous livelihood traditions, 
therefore under the framework of proper policy and guidelines, these resources can be more effectively utilized for horticultural crop improvement programmes, sustainable utilization and conservation strategies. The increased number of species at risk as a result of the changing climatic conditions will force the National Active Germplasm Sites (NAGS) which is responsible for plant specific to refocus, to strengthen their conservation policies and to increase their participation in recovery programs for trait specific orchid germplasm and including threatened species. Developments of techniques using both RET and non-RET species, which may have conservation applications which concerning the initiation of plant tissue into culture, multiplication, rooting, weaning, storage (including cryopreservation), are helpful to achieve this conservation programme. Orchids should be one of the premier groups of flowering plants for evolutionary studies, and the massive amounts of DNA data now accumulating are revolutionizing our ideas about these wonderful plants. Conservation through establishing botanic gardens, orchid biosphere reserves, orchid corridors and cryopreservation will helps for sustainable utilization for future generations.

\section{Acknowledgements}

We thank the former Director, present Director, ICAR-NRCO, Dr. Rampal, Principal Scientist (Horticulture) for his encouragement and constant support. We acknowledge the permissions from State Biodiversity Boards from respective State Governments viz., Meghalaya, Arunachal Pradesh, Madhya Pradesh, Odisha and Sikkim; apart from National Biodiversity Authority, Chennai for exemption for the purpose of research in India. We also thank Dr. Dinesh Kumar Agarwala, Scientist-D, Botanical Survey of India, Regional Circle, Gangtok, Sikkim for assistance in species identification.

\section{References}

Ahmedullah, M. and M.P. Nayar (1987).Endemic Plants of the Indian region-Vol. 1. Peninsular India, Botanical Survey of India, Calcutta, 262pp.

Ansari, R. and N.P. Balakrishnan (1990). A revision of the India species Oberonia (Orchidaceae). Orchid Monographs 4: 1-82.

Barik, SK., O.N. Tiwari, D. Adhikari, P.P. Singh, R.Tiwary and S.Barua. 2018. Geographic distribution pattern of threatened plants of India and steps taken for their conservation. Current Science, Vol.114, No.3, pp 470-595.

Blatter, E. (1928). A list of orchids with some new species from High Wavy Mountain (Madurai District).Journal of the Bombay Natural History Society 32: 518-523.

Bose, T.K. and S.K. Bhattacharjee (1980). Orchids of India. Naya Prokash, Calcutta, xxiii+538pp.

Deb C. R., Deb M.S., Jamir N.S. and Imchen T. (2009) Orchids in indigenous system of medicine in Nagaland, India. Pleione. 3(2): 209 - 211.

Deb C.R. (2013). Orchids of Nagaland, propagation, conservation and sustainable utilization: a review. Pleione 7(1): 52-58

Deb C.R. and Imchen T. (2008) Orchid Diversity of Nagaland. SciChem Publishing House, Udaipur, Rajasthan, India.

Devadas, R., S. L. Pattanayak, P. Khatiwara, S. Baruah, B. Lepcha and D. Barman.(2014).Orchid Genetic Resources in India for Varietal Development. Proceedings of National Symposium on Crop Improvement for Inclusive Sustainable Development, Punjab Agricultural University, Ludhiana-141004, Punjab, India. Nov 
7 - 9, 2014, pp 868-869

Devadas, R., S. L. Pattanayak and D. R. Singh (2016). Studies on cross compatibility in Dendrobium species and hybrids. Indian J. Genet., 76(3): 344-355

Devadas, R., R.K. Pamarthi, A.L. Meitei, S.L. Pattanayak and R. Sherpa.(2019) Morphological description of Novel Phaius primary hybrid (Orchidaceae). Journal of Experimental Biology and Agricultural Sciences.Vol.7(2) : 138147.

Duggal, S. S. (1972). Orchids in human affairs. Acta. Phytother.19: $163-173$.

Fay, M. F. and Krauss, S. L. (2003) Orchid conservation genetics in the molecular age. In Orchid Conservation (Dixon, K.W. et al., eds), pp. 91-112, Natural History Publications.

Gutierrez R M P (2010) Orchids: A review of uses in traditional medicine, its phytochemistry and pharmacology. Journal of Medicinal Plant Research, 4(8): 592-638.

Hooker, J.D. (1888-1890). Flora of British India.Vols. 5 and 6. L. Reeve and Co., London. 667-858 and 1-198pp.

IPNI (2012). The International Plant Name Index. http://www.ipni.org.

IUCN Standards and Petitions Subcommittee (2011).Guidelines for Using the IUCN Red List Categories and Criteria.Version 9.0.Prepared by the Standards and Petitions Subcommittee.

Karthikeyan, S. (2000).A statistical analysis of flowering plants of India, pp. 201-217. In: Singh, N.P., P.K. Singh, P.K. Hajra and B.D. Sharma (eds.). Flora of India, Introductory Vol. 2, Botanical Survey of India, Calcutta, xi+469pp.

Kasera PK and Shukla JK (2001).Biomedicinal properties and cultivation of Leptadaenia reticualta (Jivanti) - an endangered plant of the Thar Desert, India. Curr. Sci. 84(7):877-8.
Kholia BS (2013) Occurrence of endangered orchid Cymbidium whiteae King and Pantl. in North Sikkim. Curr Sci 104(1): 12-13

King $\mathrm{G}$ and Pantling R (1898) Ann. R. Bot. Gard. Calcutta, 8: 1-342, T. 1-448.

Kumar, C.S. and K.S. Manilal (1994). A Catalogue of India Orchids. Bishen Singh Mahendra Pal Singh, Dehradun, iii+162pp.

Lucksom SZ (2007) The Orchids of Sikkim and North East Himalaya, Gangtok, pp. 1-984.

Lucksom SZ (2008) In Special Habitats and Threatened Plants of India. ENVIS Bulletin: Wildlife and Protected Areas, Vol. 11 (ed. Rawat, GS), Wildlife Institute of India, Dehradun, Pp. 6367.

Lucksom SZ (2011) In Biodiversity of Sikkim: Exploring and Conserving a Global Hotspot (eds Arrawatia ML, Tambe S), Information and Public Relations Department, Govt of Sikkim, Pp. 125150.

Mabberley DJ (2008) Mabberley's Plantbook, A Portable Dictionary of Plants, their Classifications and Uses. 3rd Edition. University of Washington Botanic Gardens, Seattle.

Medhi, R.P. and Chakrabarti, S. (2009). Traditional knowledge of NE people on conservation of wild orchids. Indian J. Trad.Knowl. 8:11 - 16.

Meitei AL., Kiran Babu, P and Singh D.R. 2019. Orchids of India: The Silent Treasure Distribution, Utilization and Conservation. In Wild Ornamental Plants of India - Khuraijam JS (Ed.) Astral International Publishing Company, New Delhi. Pp. 277-292.

Meitei AL., Pamarthi RK., Raj Kumar, Bhutia NT., Rai D, Kiran Babu P, Singh AK, Gazmer R and Singh DR. 2019. Dendrobium nobile Lindl. In Indian Traditional medicine. A phytochemical 
approach. Indian Journal of Horticulture.Vol.76 (3).

Nayar, M.P. (1996). Hot Spots of Endemic Plants of India, Nepal and Bhutan. Tropical Botanic Garden and Research Institute, Thiruvananthapuram, 252pp.

Pearce NR, Cribb PJ (2002) Flora of Bhutan: The orchids of Bhutan. In Flora of Bhutan Vol.3, Part 3 Royal Botanical Garden, Edinburg and Royal Government of Bhutan.pp.266-267.

Pradhan, U.C. (1976). Indian Orchids: Guide to Identification and Culture 1. Kalimpong. Premulaceae Books, $188 \mathrm{pp}$.

Rampal and Singh DR (2016) Endemic orchids of North East India. In: Bioprospecting of Indigenous Bioresources of North-East India.pp 31-44.

Rao, T.A. (1998). Conservation of Wild Orchids of Kodagu in the Western Ghats. The Karnataka Association for the Advancement of Science, Bangalore, 242pp.

Singh S.K., Agarwala D.K., Jalal J.S., Dash
S.S., Mao A.A., and Singh P. (2019). Orchids of India, A Pictorial Guide, Botanical Survey of India, Kolkata. $548 \mathrm{pp}$.

Singh, D.R., Pamarthi, R.K., Raj Kumar, Rai, D., Meitei, A.L. and Kiran Babu, P. 2019. Traditional artifacts form dried leaves of Cymbidium species (Orchidaceae) in Sikkim, India. Indian Journal of Traditional Knowledge. 18 (2): 390-394.

Singh, K.P., S. Phukan and P. Bujarbarua (2001).Orchidaceae, pp. 1735-1846. In: Singh, N.P. and D.K. Singh (eds.). Floristic Diversity and Conservation Strategies in India-Vol. 4.Botanical Survey of India, Kolkata.vii+2340pp.

Uniyal MR (1975). Astavarga Sandigdha Vanushadhi. Dhanawantri Partrika. Sri Jwala Ayurevd Bhawan Aligarh.

Zaman, Q F. et al., (1998) Molecular techniques employed in the assessment of genetic diversity: a review focusing on orchid conservation. Lindleyana 13:259-283.

\section{How to cite this article:}

Pamarthi, R. K., R. Devadas, Raj kumar, D. Rai, P. Kiran Babu, A. L. Meitei, L. C. De, S. Chakrabarthy, D. Barman and Singh, D. R. 2019. PGR Diversity and Economic Utilization of Orchids. Int.J.Curr.Microbiol.App.Sci. 8(10): 1865-1887.

doi: https://doi.org/10.20546/ijcmas.2019.810.217 